\title{
On Designing Tattoo Registration and Matching Approaches in the Visible and SWIR Bands
}

\author{
Xuan Xu
}

Follow this and additional works at: https://researchrepository.wvu.edu/etd

\section{Recommended Citation}

Xu, Xuan, "On Designing Tattoo Registration and Matching Approaches in the Visible and SWIR Bands" (2016). Graduate Theses, Dissertations, and Problem Reports. 6988.

https://researchrepository.wvu.edu/etd/6988

This Thesis is protected by copyright and/or related rights. It has been brought to you by the The Research Repository @WVU with permission from the rights-holder(s). You are free to use this Thesis in any way that is permitted by the copyright and related rights legislation that applies to your use. For other uses you must obtain permission from the rights-holder(s) directly, unless additional rights are indicated by a Creative Commons license in the record and/ or on the work itself. This Thesis has been accepted for inclusion in WVU Graduate Theses, Dissertations, and Problem Reports collection by an authorized administrator of The Research Repository @ WVU. For more information, please contact researchrepository@mail.wvu.edu. 


\title{
On Designing Tattoo Registration and Matching Approaches in the Visible and SWIR Bands
}

\author{
Xuan Xu \\ Thesis submitted to the \\ Benjamin M. Statler College of Engineering and Mineral Resources \\ at West Virginia University
}

in partial fulfillment of the requirements for the degree of

\author{
Master of Science \\ in \\ Electrical Engineering \\ Thirimachos Bourlai, Ph.D., Chair \\ Jeremy Dawson, Ph.D. \\ Yuxin Liu, Ph.D. \\ Lane Department of Computer Science and Electrical Engineering \\ Morgantown, West Virginia \\ 2016
}

Keywords: Biometrics, Tattoo Recognition, Multispectral Imaging, SWIR Band, Soft Biometrics, Photometric Normalization, Tattoo Registration

Copyright 2016 Xuan Xu 


\begin{abstract}
On Designing Tattoo Registration and Matching Approaches in the Visible and SWIR Bands
\end{abstract}

\author{
Xuan Xu
}

Face, iris and fingerprint based biometric systems are well explored areas of research. However, there are law enforcement and military applications where neither of the aforementioned modalities may be available to be exploited for human identification. In such applications, soft biometrics may be the only clue available that can be used for identification or verification purposes. Tattoo is an example of such a soft biometric trait. Unlike face-based biometric systems that used in both same-spectral and cross-spectral matching scenarios, tattoo-based human identification is still a not fully explored area of research. At this point in time there are no pre-processing, feature extraction and matching algorithms using tattoo images captured at multiple bands. This thesis is focused on exploring solutions on two main challenging problems. The first one is cross-spectral tattoo matching. The proposed algorithmic approach is using as an input raw Short Wave Infrared (SWIR) band tattoo images and matches them successfully against their visible band counterparts. The SWIR tattoo images are captured at $1100 \mathrm{~nm}, 1200 \mathrm{~nm}, 1300 \mathrm{~nm}, 1400 \mathrm{~nm}$ and $1500 \mathrm{~nm}$. After an empirical study where multiple photometric normalization techniques were used to preprocess the original multi-band tattoo images, only one was determined to significantly improve cross-spectral tattoo matching performance. The second challenging problem was to develop a fully automatic visible-based tattoo image registration system based on SIFT descriptors and the RANSAC algorithm with a homography model. The proposed automated registration approach significantly improves the operational cost of a tattoo image identification system (using large scale tattoo image datasets), where the alignment of a pair of tattoo images by system operators needs to be performed manually. At the same time, tattoo matching accuracy is also improved (before vs. after automated alignment) by $45.87 \%$ for the NIST-Tatt-C database and $12.65 \%$ for the WVU-Tatt database. 


\section{Acknowledgments}

First of all, I would like to express my deepest gratitude to my committee chair and advisor Dr. Thirimachos Bourlai for giving me invaluable advices, encouraging and inspiring me during the past 2 years. This thesis would not be possible without his enormous effort and help. His dedication, rigorous and diligent research attitude have guided me deeply in my whole research experience in West Virginia University. It is a great pleasure to work with him and I believe this experience will help me a lot in my future research career.

I would also like to express my sincerest thanks to my committee members, Dr. Jeremy Dawson and Dr. Yuxin Liu for giving me valuable suggestions and helping me to improve this thesis. I want to have my special thanks to Dr. Dawson for leading and overseeing the data collection effort for this tattoo project.

I would like to extend my gratitude to all my lab mates, Neeru, Michael, Yixin, Nnmadi, Rohitha and Erin, and all my friends, for their generous help and support.

Finally, I would like to thank my family, especially my parents, fiancée and sister who unconditionally love, support and understand me. 


\section{Contents}

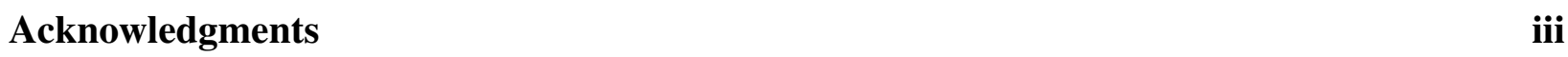

List of Figures

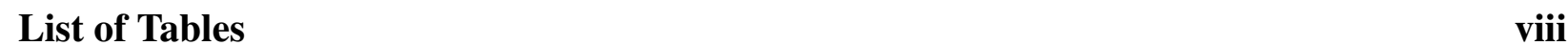

\begin{tabular}{ll}
\hline List of Abbreviations & ix
\end{tabular}

\begin{tabular}{lll|}
\hline 1 & Introduction & 1
\end{tabular}

1.1 Motivation . . . . . . . . . . . . . . . . . . 2

1.1 .1 Short-Wave Infrared . . . . . . . . . . . . . . . . . . 3

1.2 Problem Statement . . . . . . . . . . . . . . . . . . . . . . 7

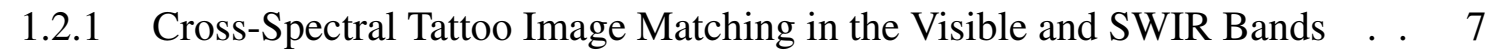

1.2.2 Tattoo Image Registration in the Visible Band . . . . . . . . . . . . . . 7

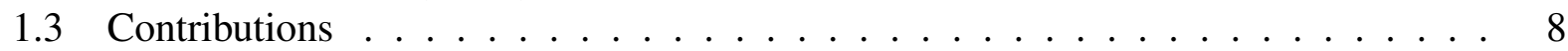

1.4 Thesis Structure $\ldots \ldots \ldots \ldots \ldots$. . . . . . . . . . . . . . . . 8

\begin{tabular}{|lll}
\hline & Related Works & 9
\end{tabular}

2.1 Tattoo Image Recognition and Retrieval . . . . . . . . . . . . . . . . . . . . 9

2.1 .1 Tattoo Image Matching . . . . . . . . . . . . . . . . . . . . . . . 99

$2.1 .2 \quad$ Mixed Media based Tattoo Image Matching . . . . . . . . . . . . . . . . . . . 11

2.2 Tattoo Localization and Segmentation . . . . . . . . . . . . . . . . . . 12

2.3 Multi-Wavelength Image Acquisition System . . . . . . . . . . . . . . . . . . . 13

$2.4 \quad$ Image Registration Approaches . . . . . . . . . . . . . . . . . . . . . . . . . . . . 14

$3 \quad$ Data Collection Setup and Experimental Design 16

3.1 Device Information . . . . . . . . . . . . . . . . . . . . 16

3.2 Experimental Setup . . . . . . . . . . . . . . . . . . . . . . . . . . . . . . . . 20

$3.2 .1 \quad$ Cross-Spectral Tattoo Matching . . . . . . . . . . . . . . . 20

3.2 .2 Tattoo Image Registration $\ldots \ldots \ldots \ldots$. . . . . . . . . . 20

\begin{tabular}{|lll}
\hline & Methodological Approach & $\mathbf{2 2}$
\end{tabular}

4.1 Cross-spectral Tattoo Matching . . . . . . . . . . . . . . . . . . . . . 22

4.1 .1 Geometric Normalization . . . . . . . . . . . . . . . . . . 22

4.1 .2 Photometric Normalization . . . . . . . . . . . . . . . . . 23 
$4.1 .3 \quad$ Feature Extraction and Matching . . . . . . . . . . . . . . . 26

4.2 Automatic Tattoo Image Registration System . . . . . . . . . . . . . . . . . 32

4.2.1 Tattoo Image Registration with SIFT Descriptor and RANSAC Algorithm . 32

$4.2 .2 \quad$ Registration Evaluation . . . . . . . . . . . . . . . . 34

4.2 .3 Registration Error Correction $\ldots \ldots \ldots$. . . . . . . . . 35

5 Experimental Results of Tattoo Matching and Registration $\quad 39$

$5.1 \quad$ SIFT vs. LBP Descriptors . . . . . . . . . . . . . . . . . . . . . . . 39

5.2 Exploring Photometric Normalization Techniques . . . . . . . . . . . . . . . . 40

5.3 Intra-spectral Tattoo Image Matching . . . . . . . . . . . . . . . . . . . 45

5.4 Tattoo Registration Results $\ldots \ldots \ldots$. . . . . . . . . . . . . . . . 46

6 Conclusions and Future Work $\quad 50$

6.1 Discussion and Conclusions . . . . . . . . . . . . . . . . 50

6.1 .1 Cross-Spectral Tattoo Matching . . . . . . . . . . . . . . . 51

6.1 .2 Tattoo Image Registration $\ldots \ldots \ldots \ldots$. . . . . . . . . . . 52

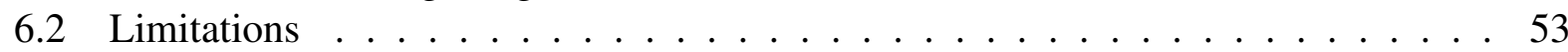

6.3 Future Work . . . . . . . . . . . . . . . . . . . . . . . 54

\begin{tabular}{ll}
\hline Bibliography & 56
\end{tabular} 


\section{List of Figures}

1.1 The general flowchart of the traditional biometric system. . . . . . . . . . . . . . 1

1.2 A masked terrorist with a tattoo on his arm [1]. $\ldots \ldots \ldots \ldots$

1.3 The example shows where the tattoo inks are in human skin and how the tattoo needle works $[2] . \ldots \ldots \ldots \ldots \ldots \ldots \ldots$

1.4 The sample tattoo images for each visible and SWIR bands. . . . . . . . . . . . . 5

1.5 The absorption curve of wavelength in water. In the range of SWIR, the absorption peak is around $1450 \mathrm{~nm}[3] . \ldots \ldots \ldots 6$

1.6 (a) shows the light intensity of tungsten light source and (b) shows the light intensity of fluorescent light source $[4] . \ldots \ldots \ldots \ldots$. . . . . . . . . 6

2.1 The overall approach flowchart of the CBIR system for tattoo image matching and

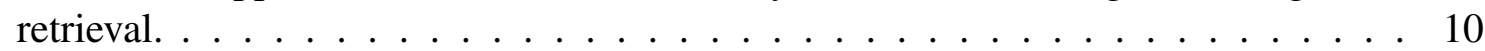

2.2 The samples of a pair of real vs. their drawn tattoo image counterparts. . . . . . . . 12

2.3 The SSMW system flowchart [4] . . . . . . . . . . . . . . . . . . . 14

3.1 Canon EOS 5D Mark III with 24-105 mm lens. . . . . . . . . . . . . . . . 17

3.2 Goodrich 1280JSX SWIR Camera $[5] . \ldots \ldots \ldots \ldots$. . . . . . . . . . 17

3.3 The collection device setup, Canon 5D Mark III, Goodrich IR camera and 5 position rotating filter wheel with 5 different wavelength filters. . . . . . . . . . . . 18

3.4 The overview of data collection setup for SWIR and visible bands tattoo image

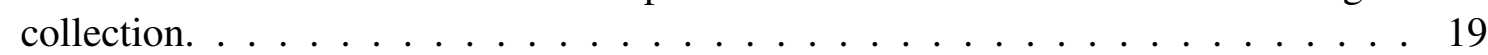

4.1 Overview of the tattoo rotation and cropping process. . . . . . . . . . . . . 23

4.2 The results of photometric normalization. The red box shows the Tan and Triggs result which we selected for our entire set of experiments. . . . . . . . . . . 27

4.3 The results of one of the photometric normalization methods, TT method is applied for all bands of SWIR and visible tattoo image . . . . . . . . . . . . . 28

4.4 A demonstration of how a basic LBP operator works. . . . . . . . . . . . . . . . 28

4.5 The example of circular LBP operator, the neighborhood points and radius are different, such as $(8,1),(8,2)$ and $(16,2)$, by bilinear interpolated. . . . . . . . . . 29

4.6 The overall approach flowchart of our proposed two-level based automatic tattoos registration system. Block (1b) indicates the detailed level one registration process of block (1a) applied SIFT and RANSAC with homography model. Block (2) shows the general registration process of level two (see Fig. 4.8 for more details about level two process). . . . . . . . . . . . . . . 33 
4.7 The general process of score fusion based image quality index technique. . . . . . 35

4.8 The general process of second level based tattoo registration system. The upper block shows an example of incorrect registration makes by level one of our system. The lower block indicates the result of level two registration after applying redchannel-CLAHE for the input pair tattoos. . . . . . . . . . . . . . 36

4.9 Tattoo Image analysis of SIFT matching for all grayscale (A), red-channel CLAHE (B), green-channel CLAHE (C) and blue-channel CHALHE (D). . . . . . . . . . . 37

5.1 The CMC curve of cross-spectral tattoo matching applied for LBP method measured by Chi squared. . . . . . . . . . . . . . . . . . . 40

5.2 The CMC curve of cross-spectral tattoo matching applied for SIFT method measured by Euclidean distance. . . . . . . . . . . . . . . . . . . . 41

5.3 The results for $1100 \mathrm{~nm}$ vs. gallery tattoo matching applied for each of the 21 photometric normalization techniques. . . . . . . . . . . . 42

5.4 The results for $1200 \mathrm{~nm}$ vs. gallery tattoo matching applied for each of the 21 photometric normalization techniques. . . . . . . . . . . . . 42

5.5 The results for $1300 \mathrm{~nm}$ vs. gallery tattoo matching applied for each of the 21 photometric normalization techniques. . . . . . . . . . . . . . 42

5.6 The results for $1400 \mathrm{~nm}$ vs. gallery tattoo matching applied for each of the 21 photometric normalization techniques. . . . . . . . . . . . . 43

5.7 The results for $1500 \mathrm{~nm}$ vs. gallery tattoo matching applied for each of the 21 photometric normalization techniques. . . . . . . . . . . . . 43

5.8 The results for all $1100 \mathrm{~nm}$ to $1500 \mathrm{~nm}$ vs. gallery tattoo matching applied for TT photometric normalization technique. . . . . . . . . . . . . 44

5.9 The results for all $1100 \mathrm{~nm}$ to $1500 \mathrm{~nm}$ vs. gallery tattoo matching applied for DoG photometric normalization technique. . . . . . . . . . . . . . 44

5.10 The results for inter and intra-spectral tattoo matching applied for TT photometric

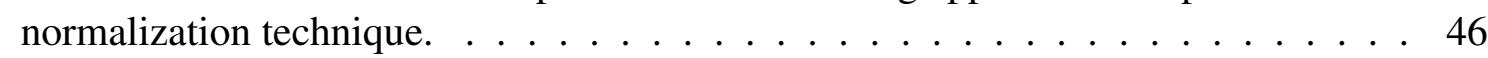

5.11 The example for already registered pair of tattoos $\ldots \ldots$. . . . . . . . . 47

5.12 CMC curves of tattoo recognition performance based on LBP with uniform patterns: (a) indicates the results of NIST-Tatt-C database; (b) indicates the results of WVU-Tatt database. . . . . . . . . . . . . . . . . 48

6.1 The comparative example for different color channels of tattoo processed by CLAHE or not. Row 1 shows the original grayscale, red, blue and green channel tattoo images. Row 2 shows the histograms of each of the images at row 1. Row 3 displays the tattoo images processed by CLAHE and row 4 shows the histograms of each of the images at row $3 . \ldots \ldots \ldots \ldots \ldots \ldots$ 


\section{List of Tables}

2.1 Summarized of tattoo image research focuses in the literatures, including pre-

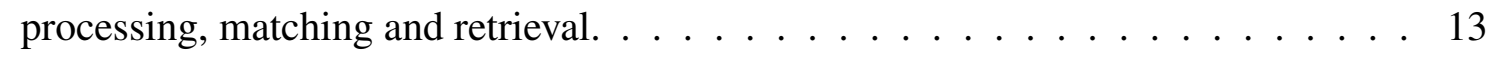

3.1 The summary of each database setup for the experiments of cross-spectral matching. 20

3.2 The summary of each database setup for the experiments of tattoo registration . . . 21

4.1 Summary of R, G, B channel combined with CLAHE for all the incorrectly registered subjects for both databases (NIST-Tattoo-C and WVU-Tatt). Numbers outside the parenthesis represent the number of SIFT keypoint matching, numbers inside the parenthesis represent the correct SIFT keypoint matching. . . . . . . . . 38

5.1 Summarized of cross-spectral matching results of each band with or without applying photometric normalization technique. . . . . . . . . . . . . 45

5.2 Results of tattoo registration accuracy including both NIST-Tatt-C and WVU-Tatt

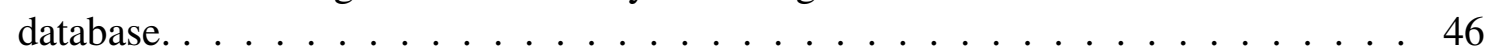

5.3 Rank-1 of tattoo recognition performance of NIST-Tatt-C and WVU-Tatt Database based on LBP method with uniform patterns. . . . . . . . . . . . . . . . . 47 


\section{List of Abbreviations}

SIFT Scale-Invariant Feature Transform

LBP Local Binary Patterns

SWIR Short-Wave Infrared

CBIR Content-Based Image Retrieval

MHLC Multiple Different Sized-bin Polar Histograms based Local Shape Context

LSS Local Self Similarity

SURF Speeded Up Robust Features

SC Shape Context

CPD Coherent Point Draft

SSMW Single-Sensor Multi-Wavelength

NIR Near-Infrared

SSR Single Scale Retinex

ASR Adaptive Single Scale Retinex

HOMO Homomorphic Filtering based Normalization

SSQ Single Scale Self Quotinet

MSQ Multi Scale Self Quotinet

DCT Discrete Cosine Transform based Normalization 
WA Wavelet based Normalization

WD Wavelet Denoising based Normalization

IS Isotropic Diffusion based Normalization

SF Steerable Filter based Normalization

NLM Non-local Means based Normalization

ANL Adaptive Non-local Means based Normalization

MAS Modified Anisotropic Diffusion Normalization

GF Gradientfaces Normalization

SSW Single Scale Weberfaces Normalization

MSW Multi scale Weberfaces normalization

LSSF Large- and small-scale Features Normalization

TT Tan and Triggs Normalization

DoG DoG Filtering based Normalization

CLAHE Contrast-Limited Adaptive Histogram Equalization

CMC Cumulative Match Characteristic

IR Infrared

SMT Scars, Marks or Tattoos

AS Anisotropic Diffusion based Normalization

RANSAC Random Sample Consensus

UIQ Universal Image Quality

FSIM Feature Similarity Image Quality

SAR Synthetic Aperture Radar 


\section{Chapter 1}

\section{Introduction}

Biometrics is the science of using physiological or behavioral characteristics, such as face, iris, fingerprint, voice, gait and signature, to identify or verify individuals [6]. A traditional biometric recognition system is composed of a set of modules, as illustrated in Fig. 1.1, which can be summarized as follows: (1) sensors are used to capture data; (2) data are captured by sensors to input the recognition system; (3) the pre-processing step is applied to remove useless information or enhance local and global features of input data; (4) the feature extractor is implemented to extract unique features from input data; (5) through the matcher, the probe dataset can be matched with the gallery dataset to achieve the goal of identification or verification purposes.

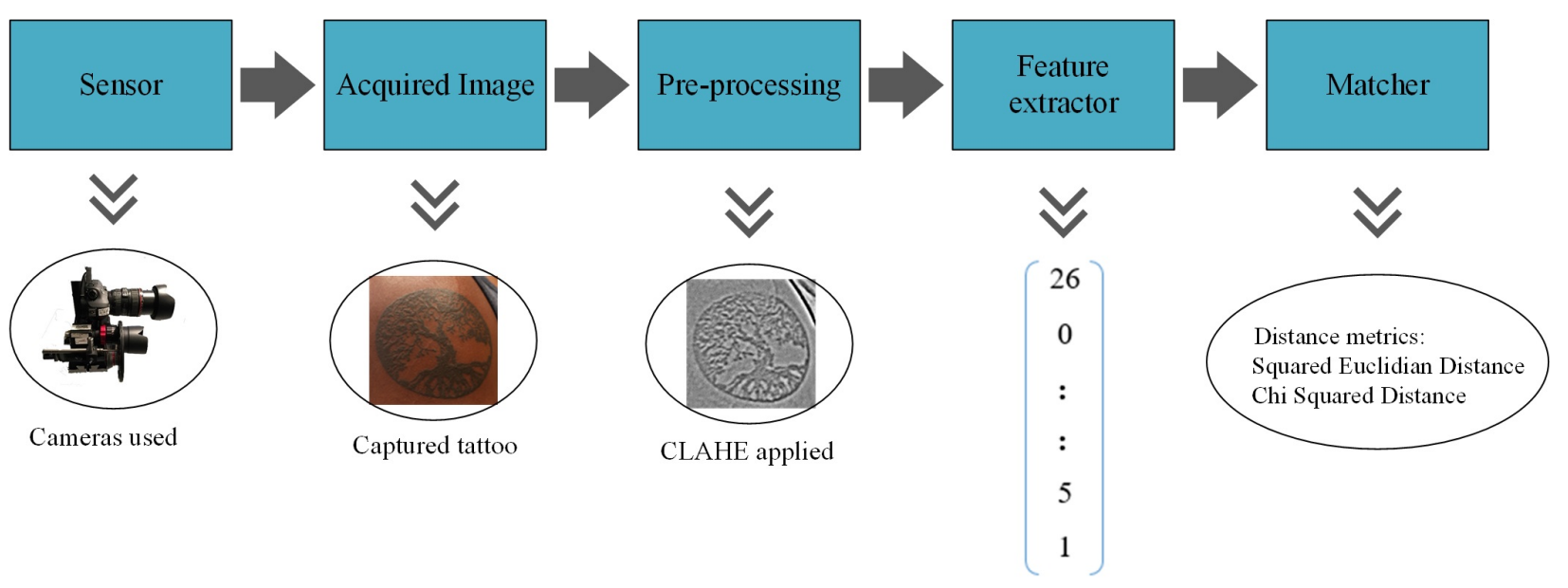

LBP feature vector

Figure 1.1: The general flowchart of the traditional biometric system. 


\subsection{Motivation}

Aside from conventional biometric traits, soft biometric traits have been considered as alternative sources of information, useful for human identification [7]. And sometimes, soft biometrics may be the only clue available that can be used for identification or verification purposes (see Fig. 1.2). In [8], Dantcheva et al. defined, "Soft biometrics are traits physical, behavioral or adhered human characteristics, classifiable in pre-defined human compliant categories. These categories are, unlike in the classical biometric case, established and time-proven by humans with the aim of differentiating individuals. In other words, the soft biometric traits instances are created in a natural way, used by humans to distinguish their peers". Soft biometrics was first presented by Alphonse Bertillon in the 19th century and also the first personal biometric identification system is designed by him [9]. Many characteristics are considered as soft biometric traits, including color of eye, hair, skin and beard; shape and size of head; height, weight, and also Scars, Marks or Tattoos (SMT) [9]. In [10], Jain et al. defined "soft biometric traits as characteristics that provide some information about the individual, but lack the distinctiveness and permanence to sufficiently differentiate any two individuals". There has been proved that it is possible to use the larger scale of soft biometric traits to achieve the identification or verification purposes. In general, surveillance applications are deployed with soft biometrics [11] and there are a lot of research efforts that capitalize on these developments. For example, the Federal Bureau of Investigation (FBI) Next Generation Identification (NGI) system uses the soft biometric traits like SMT as additional biometric modalities to identify criminals [7] [12].

Among the multifarious soft biometric traits, tattoo has been identified in practical biometric applications to be a very important evidence for human identification. Tattoo images, when captured under certain conditions (e.g. when the images are not affected by extreme pose, illumination variations and low quality) can be more useful to distinguish different individuals than other traditional demographic indicators, such as height, gender, age and race [13]. Tattoos have been first created over 5,000 years ago [14]. The harris poll points out that nowadays tattoos become more and more prevalent in modern society. 3 out of 10 adults in United States have at least one tattoo in 2016. This number was 2 out of 10 in 2012. From those people who have tattoos, there are $69 \%$ that also mentioned they have two or more tattoos in their body. Also tattoos are very 


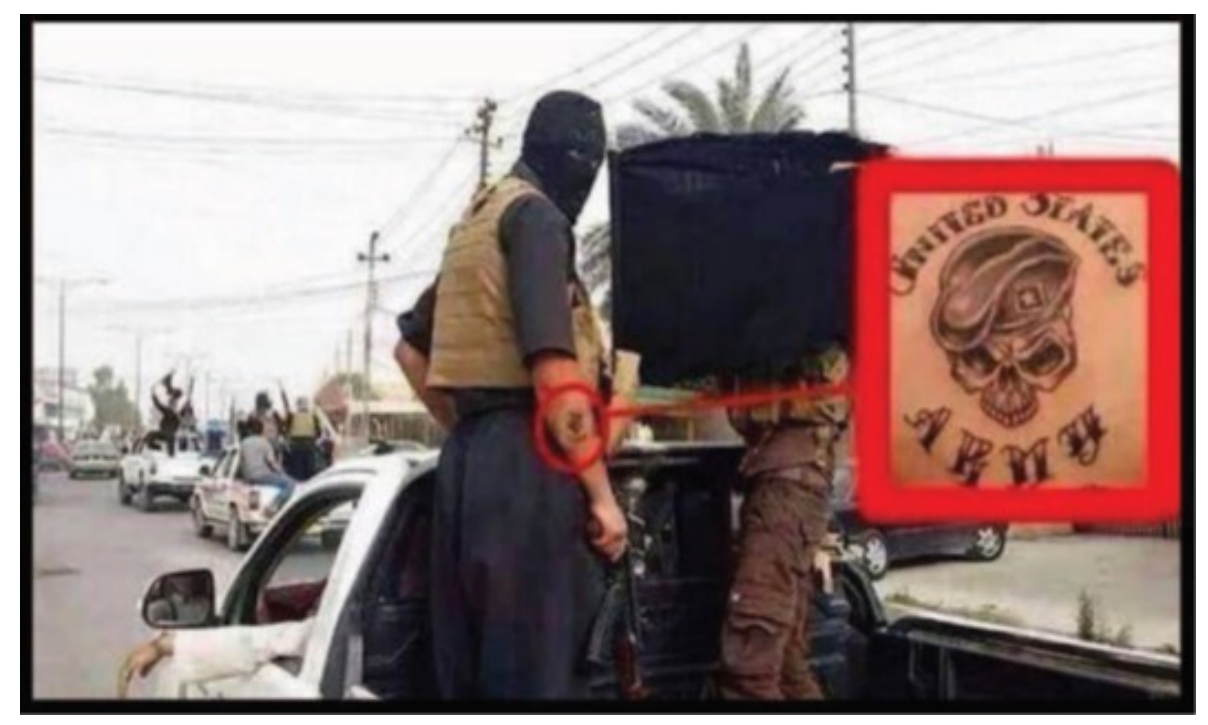

Figure 1.2: A masked terrorist with a tattoo on his arm [1].

popular among younger Americans, e.g. it is reported that about 47\% of Millennials have at least one tattoo [15].

Therefore, there are two main reasons why human tattoos can be successfully used as an alternative way of human identification in forensics. The first one is that tattoos have become more and more popular, and the second one is that tattoos have already influenced other human identification methods such as pathological, visual and trauma-based identification [16]. An important feature of human tattoos is that tattoo pigments are found deeply in the human skin so that they can be preserved even under skin burns. A practical example of why tattoos are important in human identification is the fact that they were used for victim identification in the 9/11 terrorist attacks in 2001 and the Asian tsunami tragic event in 2004 [13].

\subsubsection{Short-Wave Infrared}

Short-Wave Infrared (SWIR) has been widely used in face recognition systems. Beyond the visible band, SWIR can be used to capture image data in harsh environment such as in fog, smoke and at nighttime. This feature of SWIR may help surveillance system to collect high quality image at crime scene under severe conditions. However, in the open literature, no tattoo recognition system based on SWIR tattoo dataset is explored.

In this thesis, we collected visible and SWIR tattoo databases, also implemented cross-spectral 


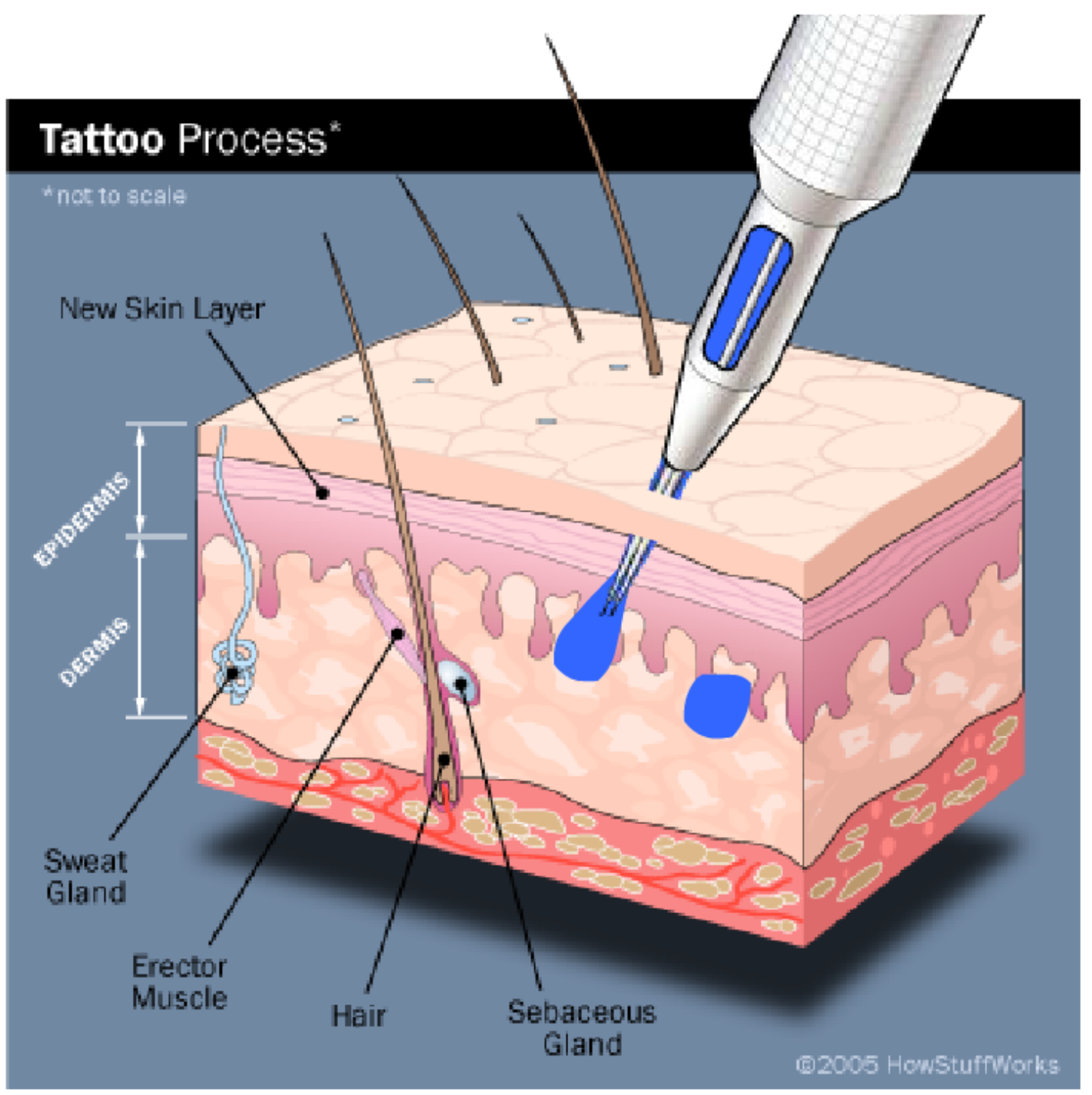

Figure 1.3: The example shows where the tattoo inks are in human skin and how the tattoo needle works [2]. 

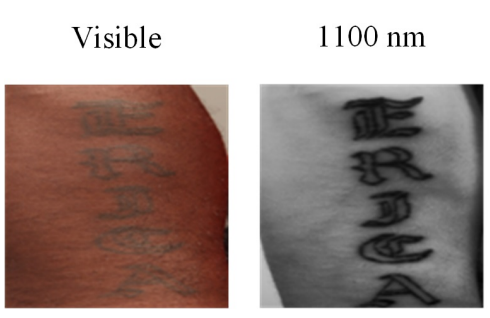

$1200 \mathrm{~nm}$

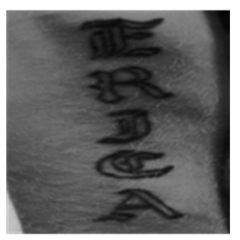

$1300 \mathrm{~nm}$

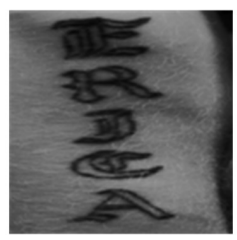

$1400 \mathrm{~nm}$

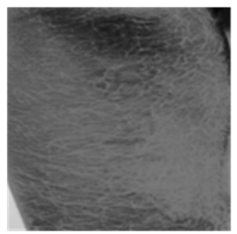

$1500 \mathrm{~nm}$

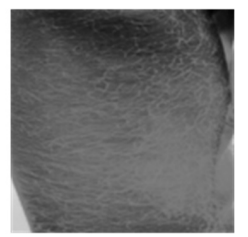

Figure 1.4: The sample tattoo images for each visible and SWIR bands.

and same-spectral tattoo image matching. As we know, tattoo is placed in human skin. Human skin is constructed by three layers, epidermis, dermis and hypodermis. According to [17], tattoo inks should be input into the dermis layer by a tattoo needle. Basically, the depth of tattoo inks is around $1 \mathrm{~mm}$ to $2 \mathrm{~mm}$ (see Fig. 1.3). Since the penetrability of SWIR (in general, SWIR can penetrate into skin around $2.5 \mathrm{~mm}$ to $5 \mathrm{~mm}$ [18]) is better than visible light, the tattoo image collected by SWIR camera has a clearer contour than the image collected by visible band camera (See Fig. 1.4, regarding the tattoo images of visible, $1100 \mathrm{~nm}, 1200 \mathrm{~nm}$ and $1300 \mathrm{~nm}$ ). However, in Fig. 1.4, the tattoo images captured from $1400 \mathrm{~nm}$ and $1500 \mathrm{~nm}$ bands are suffering from bad quality issue. This is a big challenge for tattoo recognition system. The moisture content of the dermis is around 60-70\% [19], and according to Fig. 1.5, the water absorption peak of SWIR is around $1450 \mathrm{~nm}$. Therefore, when we used the bands of $1400 \mathrm{~nm}$ and $1500 \mathrm{~nm}$ to capture tattoo images, most of SWIR was absorbed by the water in dermis, the skin became dark and the quality of tattoos was decreased.

Another important factor may influence the SWIR tattoo data collection is the light source used. According to the work presented by Bourlai et al. [4], tungsten light source has the better intensity for the SWIR than the traditional fluorescent light source. Fig. 1.6 shows the light intensity of both tungsten and fluorescent light sources in the wavelength range from $900 \mathrm{~nm}$ to $1600 \mathrm{~nm}$. The Fig. 1.6 (a) indicates the intensity of tungsten light source. From the response curve of tungsten light source, the light intensity is stable, and covering all of the wavelengths from $1100 \mathrm{~nm}$ to 1500 nm. The Fig. 1.6 (b) demonstrates the intensity of fluorescent light source. From the response curve of fluorescent light source, the light intensity has very limited response for the wavelengths we used in this work $(1100 \mathrm{~nm}, 1200 \mathrm{~nm}, 1300 \mathrm{~nm}, 1400 \mathrm{~nm}$ and $1500 \mathrm{~nm})$. Therefore, we selected tungsten light source for the SWIR tattoo data collection. 


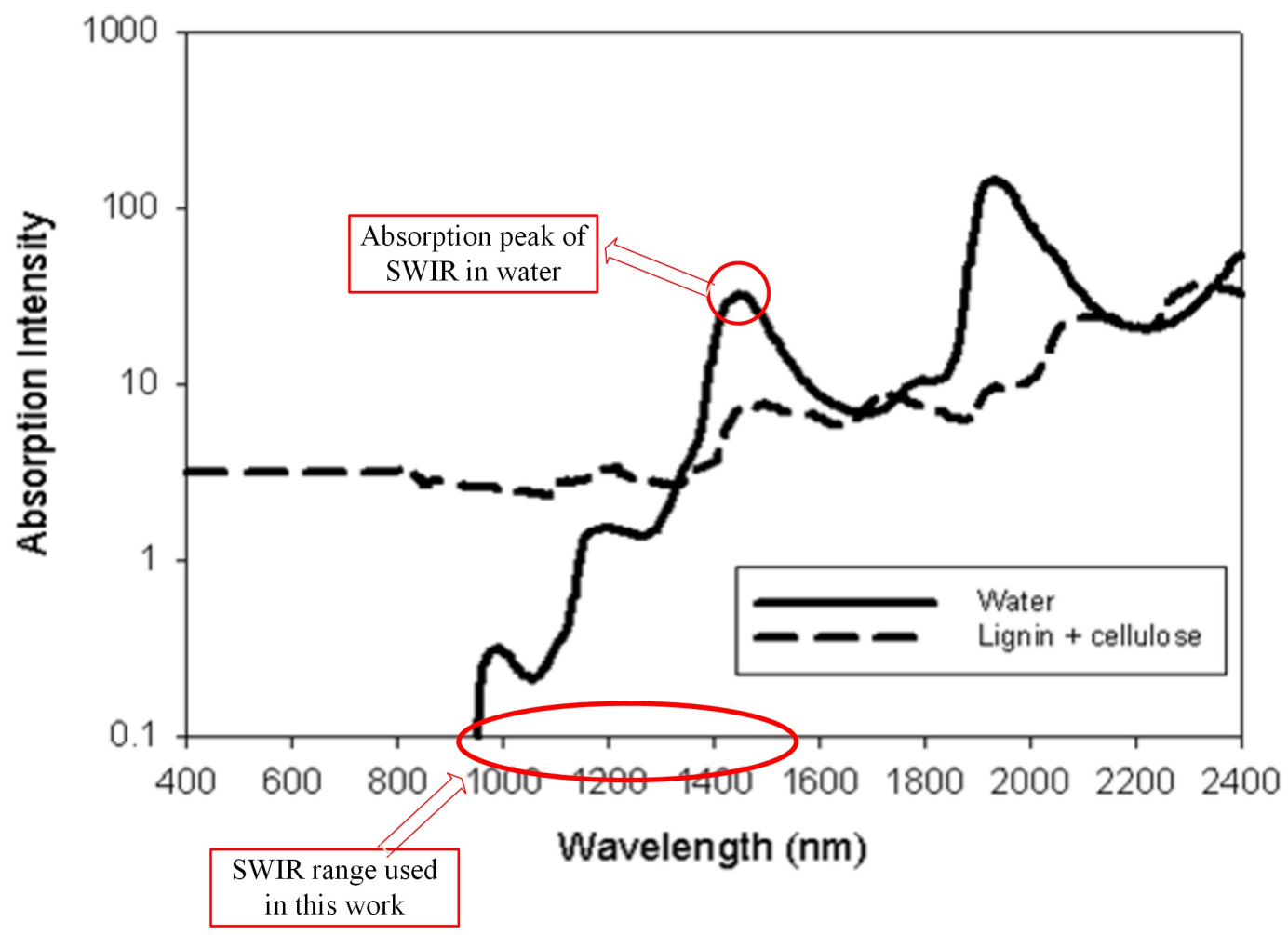

Figure 1.5: The absorption curve of wavelength in water. In the range of SWIR, the absorption peak is around $1450 \mathrm{~nm}[3]$.

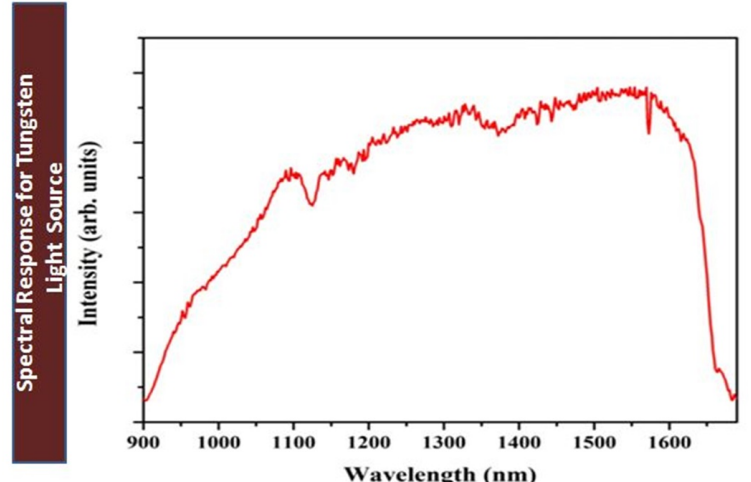

(a)
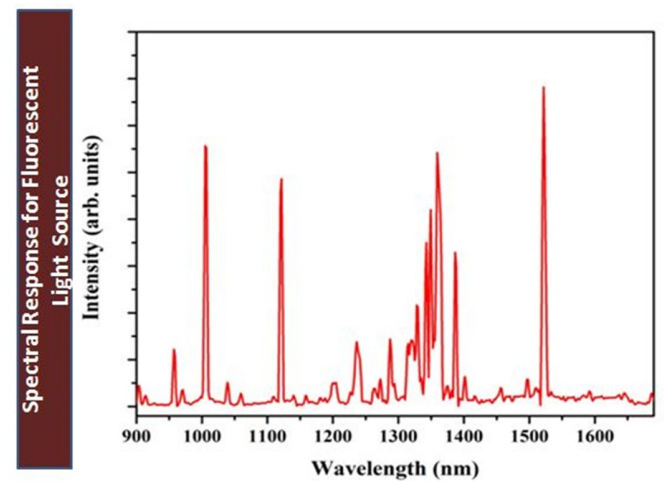

(b)

Figure 1.6: (a) shows the light intensity of tungsten light source and (b) shows the light intensity of fluorescent light source [4]. 


\subsection{Problem Statement}

In this thesis, we mainly focus on solving two main challenging problems: 1) Cross-spectral tattoo image matching using visible and SWIR band images; 2) Tattoo image registration in the visible band.

\subsubsection{Cross-Spectral Tattoo Image Matching in the Visible and SWIR Bands}

In this problem, we collected the visible and SWIR tattoo image databases. Each database is composed of a set of visible and SWIR datasets. For the SWIR dataset, 5 bands of SWIR filters are used $(1100 \mathrm{~nm}, 1200 \mathrm{~nm}, 1300 \mathrm{~nm}, 1400 \mathrm{~nm}$ and $1500 \mathrm{~nm})$. Then, an experiment has been implemented to select feature extractor between Scale-Invariant Feature Transform (SIFT) [20] and Local Binary Patterns (LBP) [21]. Moreover, we explored whether certain photometric normalization methods, can, first, enhance the features of both SWIR and visible tattoo images and then, improve cross-spectral matching performance. Finally, we investigated whether intraspectral matching is also improved when using the selected feature descriptor and the most efficient photometric normalization method in terms to its effect in matching accuracy.

\subsubsection{Tattoo Image Registration in the Visible Band}

A two-level based automated registration system is designed and developed by using SIFT descriptors and the Random Sample Consensus (RANSAC) [22] algorithm with a homography model. The first level of our system uses a traditional SIFT registration routine with the RANSAC method to filter outliers of SIFT matchers. In the evaluation mode, a score level fusion scheme of the Universal Image Quality (UIQ) [23] and Feature Similarity Image Quality (FSIM) [24] indexes is used to decide whether a pair of tattoo images is registered correctly or not before matching is performed. Our system will automatically make a decision whether a pair of tattoo images can be an input to the second level of our system that focused on error correction. Here, the redchannel of each RGB tattoo image is first used, and then the Contrast-Limited Adaptive Histogram Equalization (CLAHE) [25] method is applied so that the error correction level is efficient. 


\subsection{Contributions}

The main contributions of this thesis are two-fold:

1. We explored cross-spectral tattoo image matching in visible and SWIR bands, by selecting feature descriptor (SIFT) vs. LBP $)$ and photometric normalization technique through an empirical study, the matching performance of either cross-spectral or intra-spectral matching is improved significantly.

2. We designed a two-level based automated tattoo image registration approach which is associated with SIFT descriptors and the RANSAC algorithm with a homography model to register visible band tattoo images.

\subsection{Thesis Structure}

The rest of this thesis is organized as follow:

- Chapter 2 introduces the existing works and techniques related to tattoo recognition systems and image registration techniques.

- Chapter 3 presents the experimental design and data collection setup, including the detailed stages of data collection, the information of equipment and the environment of collection. Also this chapter describes the scenario of tattoo registration system.

- Chapter 4 describes the methodological approach of this thesis, including pre-processing, feature extraction, feature descriptor matching and also carrying out the approach of tattoo registration system.

- Chapter 5 provides the experimental results of cross-spectral tattoo image recognition and tattoo registration.

- Chapter 6 represents the conclusions and future work of this thesis. 


\section{Chapter 2}

\section{Related Works}

This chapter introduces the existing works related to tattoo localization, segmentation, recognition and retrieval. Also this chapter reviews the image registration techniques and developed multi-wavelength acquisition system in SWIR band which is adapted for this work to collect SWIR band tattoo images.

\subsection{Tattoo Image Recognition and Retrieval}

Tattoo image retrieval has become a new biometric research topic to identify humans in the past ten years. Researchers have proposed many outstanding works for tattoo image matching and retrieval, based on image-to-image matching and mixed media matching.

\subsubsection{Tattoo Image Matching}

Jain et al. [26] designed a Content-Based Image Retrieval (CBIR system for tattoo image matching and retrieval. In their work, firstly they used a $3 \times 3$ Sobel operator to segment the tattoo images as the preprocessing step. Then, low-level image features were extracted, such as color, shape and texture. The color features included color histogram and color correlogram [27, 28]; according to [27], the segmented grayscale tattoo images and gradient tattoo images are used to extract the shape features; to extract the texture features, they used the Edge Direction Coherence Vector [29]. In the matching approach, this work computed the similarity score by applying histogram intersection method [30] for the tattoo images. The general [CBIR system for tattoo 
matching and retrieval is displayed in Fig. 2.1.

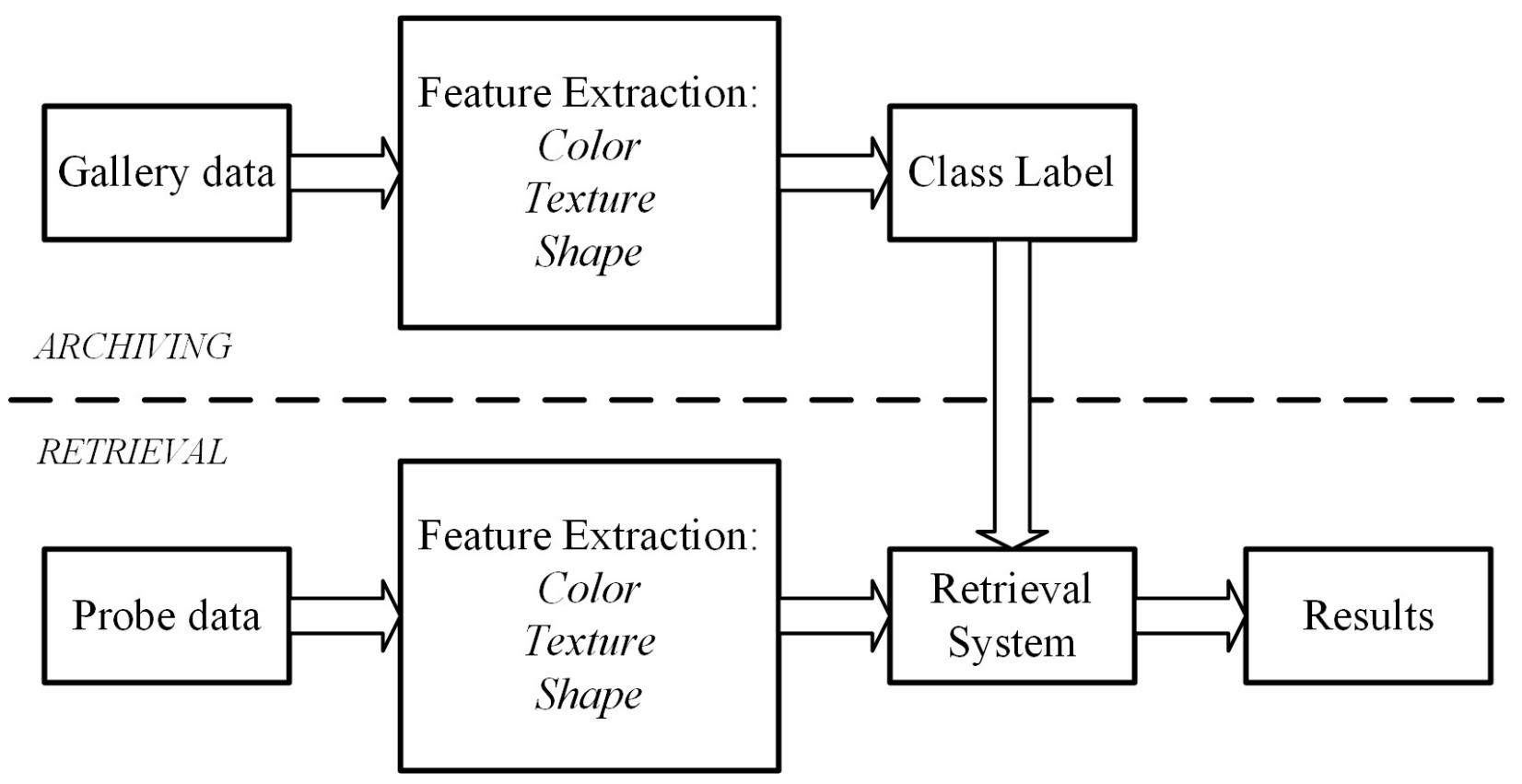

Figure 2.1: The overall approach flowchart of the CBIR system for tattoo image matching and retrieval.

After that, Lee et al. [31] proposed a new $\mathrm{CBIR}$ system for tattoo image retrieval by replacing the low-level features to the SIFT features [20]. This system ranks the number of SIFT keypoints matched of a query image with gallery images to retrieve the target tattoo image, the top retrieved image is defined as the retrieval result which has the most number of SIFT keypoints matched with the query image. Moreover, Jain et al. [32] proposed two strategies, the body location of tattoo image and geometric constraints, to improve the retrieval performance of CBIR system. For the body location approach, they classified the tattoo database based on the ANSI/NIST ITL 12000 standard [33]. For the geometric constraints approach, they separated the matched keypoints as true matching points and false matching points. A true keypoint can always match with few other keypoints and a false keypoint can always match with large numbers of other keypoints. Therefore, they only used the true matching keypoints to retrieve tattoo images. In [34], Lee et al. proposed an unsupervised ensemble ranking method to adapt the large scale database of tattoo images retrieval for CBIR system. The retrieval performance of this method is not the best one among other methods by using their CBIR system, but this one is the best trade-off method. 
It is much faster than SIFT matching [31] and the combination of ensemble and SIFT method. Although the bag-of-words model [35] is the fastest method for CBIR system, but the proposed unsupervised ensemble ranking method has better matching result and is just a little bit slower than bag-of-words model. Then, Lee et al. [36] continually improved the tattoo retrieval performance of CBIR system by enhancing the similarity measurements, including weighted SIFT keypoints matching, symmetric matching and asymmetric matching.

Kim et al. [37] proposed a new tattoo matching and retrieval approach by combining local and global matching methods. They introduced a descriptor called Multiple Different Sized-bin Polar Histograms based Local Shape Context (MHLC) to combine with SIFT descriptor as the local features. For the global shape descriptor, this work used the multiple different sized-bin polar histogram associate with 2D discrete Fourier transform operator. In [38], Kim et al. introduced another new tattoo image matching descriptor by combining SIFT descriptor and Local Self Similarity (LSS) image descriptor [39], and defined a weighted distance similarity for the tattoo image matching. Yi et al. [40] evaluated the effect of tattoo segmentation on the tattoo matching by removing the useless image background for either probe set and gallery set. In this work, four experimental settings were applied: 1) use original image to match; 2) remove background of gallery set; 3) remove background of probe set; 4) remove background of both probe and gallery sets. Two matching method were used, SIFT and SURF [41].

\subsubsection{Mixed Media based Tattoo Image Matching}

Sometimes the query tattoo image maybe not available for the surveillance system to acquire. Therefore, a drawn based tattoo query image can become a clue to identify the criminal suspect (samples of mixed media tattoo images are shown in Fig. 2.2 from [42]). There have been proposed two mixed media tattoo image based matching approaches in the past several years.

Han and Jain [43] presented to use tattoo sketch images matching with real tattoo images by applying canny edge detector [44] to extract the shape information of both and real tattoo images, and then using SIFT to extract local patterns from the shape of tattoos. This is the first time that sketch tattoo media are considered as query data to be used for tattoo human identification. After that, Huffman and McDonald [45] proposed another approach for mixed media tattoo matching 

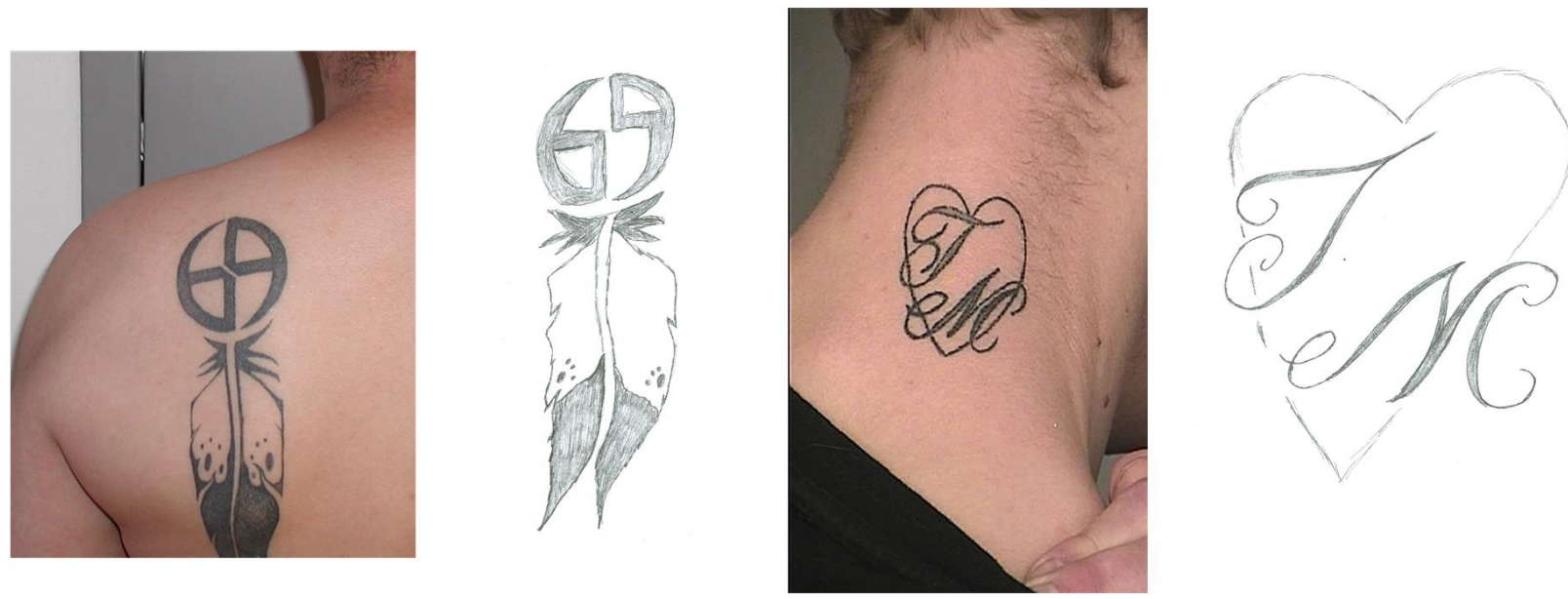

Figure 2.2: The samples of a pair of real vs. their drawn tattoo image counterparts.

based on Shape Context (SC) [46], Coherent Point Draft (CPD) [47], and [CPD mixture weights to extract edge information from sketch and real tattoo images, then by computing the score alignment quality to measure the similarity of the extracted features.

\subsection{Tattoo Localization and Segmentation}

Tattoo localization and segmentation are two very important pre-processing steps to improve the performance of tattoo matching and retrieval. Most of tattoo image matching and retrieval techniques that introduced in the previous section involved to crop region of interest (areas of tattoo located at) manually. But the work of cropping tattoos by human operators is time consuming, and inefficient. Therefore, researchers proposed several approaches to try to crop tattoos from the original images automatically. Duangphasuk and Kurutach [48] proposed an image negative method based tattoo image segmentation as a pre-processing scenario for tattoo image matching and retrieval. This method includes three steps: (1) the skin color segmentation associated with HSV (hue, saturation and value) model is used for skin detection; (2) image negative method is used for tattoo skin detection; (3) finally, tattoos are segmented from the original image based on the result getting from image negative method. Allen et al. [49] introduced another tattoo segmentation method by using a bottom-up and top-down combination method. This method is based on an unsupervised clustering to separate the skin and tattoo. Kim et al. [50] proposed two 


\begin{tabular}{|l||c|c|c|c|c|}
\hline Literature & Label & Localization & Segmentation & Matching & Retrieval \\
\hline \hline Jain et al. [26] & $\times$ & - & $\times$ & $\times$ & $\times$ \\
\hline Lee et al. [31] & $\times$ & - & - & $\times$ & $\times$ \\
\hline Jain et al. [32] & $\times$ & - & - & $\times$ & $\times$ \\
\hline Duangphasuk et al. [48] & - & - & $\times$ & $\times$ & $\times$ \\
\hline Allen et al. [49] & - & - & $\times$ & - & - \\
\hline Yi et al. [40] & - & - & - & $\times$ & - \\
\hline Kim et al. [37] & - & - & - & $\times$ & $\times$ \\
\hline Kim et al. [38] & - & - & - & $\times$ & $\times$ \\
\hline Kim et al. [50] & - & $\times$ & $\times$ & - & - \\
\hline
\end{tabular}

Table 2.1: Summarized of tattoo image research focuses in the literatures, including preprocessing, matching and retrieval.

new approaches related to automatic tattoo localization. The first method is called center-surround feature localization, which is a combination method to use center-surround filter to associate with skin color features and edge features. The second one is called graph-cut tattoo localization which is based on their previous work [51]. Another contribution of this work is that they described a tool for segmenting and saving tattoo image manually. Table 2.1 listed a summary of explored tattoo image research focuses.

\subsection{Multi-Wavelength Image Acquisition System}

Multi-Wavelength systems have been used for many research areas. One of the important use of Multi-Wavelength systems for biometrics is to obtain face images from different bands such as infrared face images and thermal face images. Here we will introduce one of the system design which has been modified and used for this work to collect our tattoo database.

Bourlai et al. [4] and Narang et al. [52] designed a Single-Sensor Multi-Wavelength (SSMW) system to obtain visible and SWIR face image database that constructed with Goodrich SU640 (SWIR Camera), Five-position Rotation Filter Wheel, DC Servo motor and Optical Reflective Sensor. The overall flowchart of their system is shown in Fig. 2.3 . The subject is sitting right in 
front of the camera, the distance between camera and subject is $2 m$ and the light source would be coming from left and right side of the subject. By changing the filter wheel, operators can collect different bands of SWIR face images including $1150 \mathrm{~nm}, 1250 \mathrm{~nm}, 1350 \mathrm{~nm}, 1450 \mathrm{~nm}$ and 1550 $n m$.

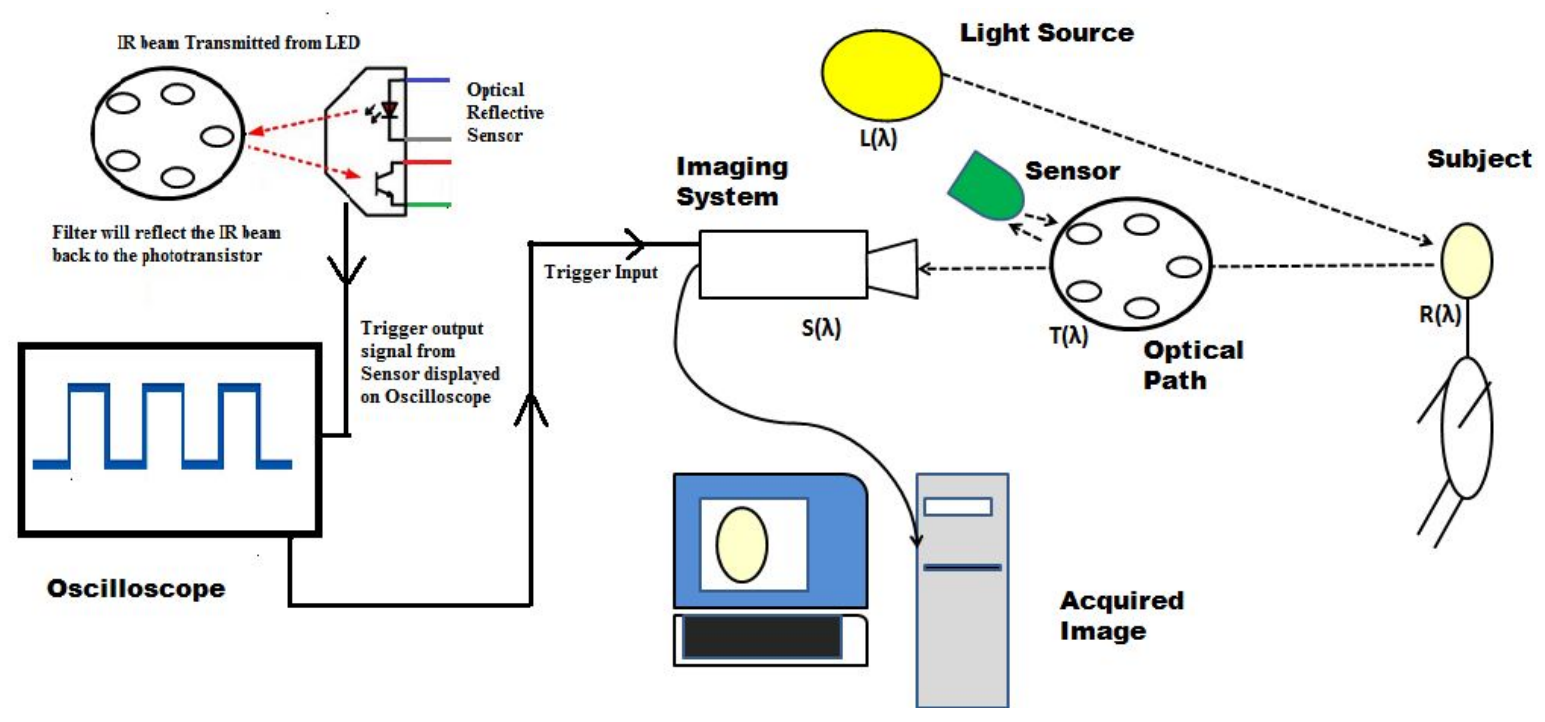

Figure 2.3: The SSMW system flowchart [4]

\subsection{Image Registration Approaches}

Image registration is the process related to align multiple images into the same coordinate system. The general process of image registration can be expressed as follow: to apply geometric transformation methods for a set of input images to align with a reference image so that they are able to locate at the same scene. In general, image registration process includes four basic steps: feature detection, feature matching, transform model estimation and image resampling and transformation [53]. Several image registration techniques have been proposed. Dare and Dowmen [54] introduced an advanced model using edge extraction and matching of Synthetic Aperture Radar (SAR] registration. Yu et al. [55] proposed another fully automatic and fast non-rigid image registration approach based on SIFT] and the Harris corner detector methods. Wong and Clausi [56] used robust complex wavelet feature representations to register satellite images by constraining 
point detection so that it can be adapted for the proposed image registration system. Although there are many image registration applications existing, to the best of our knowledge, no fully automated image registration method has been proposed for tattoo images. 


\section{Chapter 3}

\section{Data Collection Setup and Experimental Design}

This Chapter introduces: (1) the devices used to collect SWIR and visible tattoo databases; (2) the database structures and setup for both cross-spectral matching and tattoo image registration.

\subsection{Device Information}

Our data collection system consists of the following components: Canon EOS 5D Mark III Camera, Goodrich 1280JSX SWIR camera, a 5-position rotating filter wheel, and 5 different wavelength filters.

- Canon EOS 5D Mark III: The Canon 5D Mark III camera is composed by a 22.3 Megapixel full-frame Canon CMOS sensor and a Canon DIGIC 5+ image processor. This camera is designed with the features of full frame, high-resolution image capture, the 61-Point high density reticular AF and a flexible ISO range of 100-25600. The lens of this camera equipped is Canon EF 24-105 mm, f/4 maximum aperture. The Fig. 3.1 shows the camera with lens.

- Goodrich 1280JSX (SWIR Camera): Goodrich 1280JSX (see Fig. 3.2) is the next generation digital video SWIR camera yielded by Sensors Unlimited. It owns a 1.3 Megapixel high-resolution, high-sensitivity (from 700 to $1700 \mathrm{~nm}$ for Near-Infrared (NIR)/SWIR wavelength spectrum) InGaAs (Indium Gallium Arsenide) imager. Also it supports $60 \mathrm{fps}$ full 


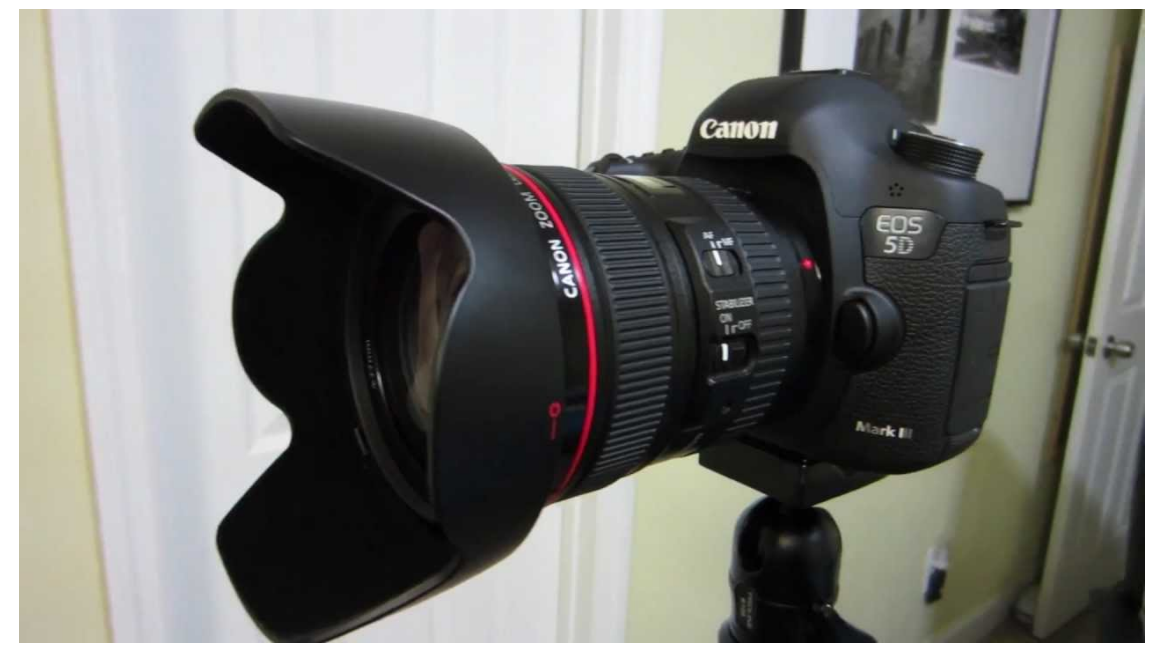

Figure 3.1: Canon EOS 5D Mark III with 24-105 mm lens.

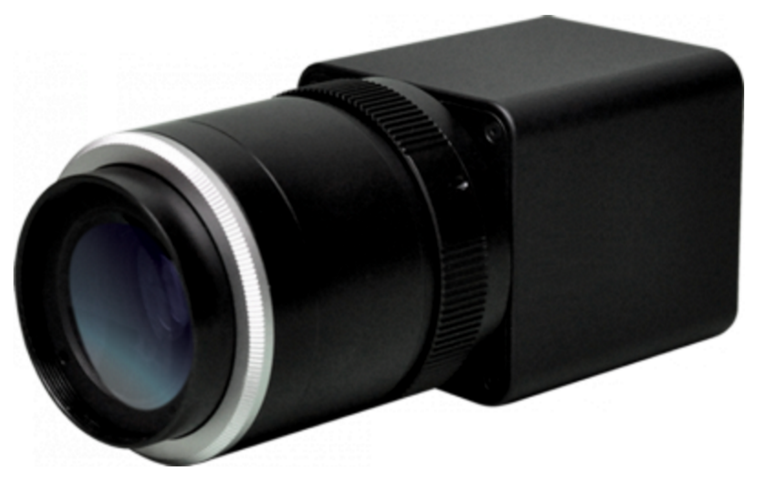

Figure 3.2: Goodrich 1280JSX SWIR Camera [5]. 

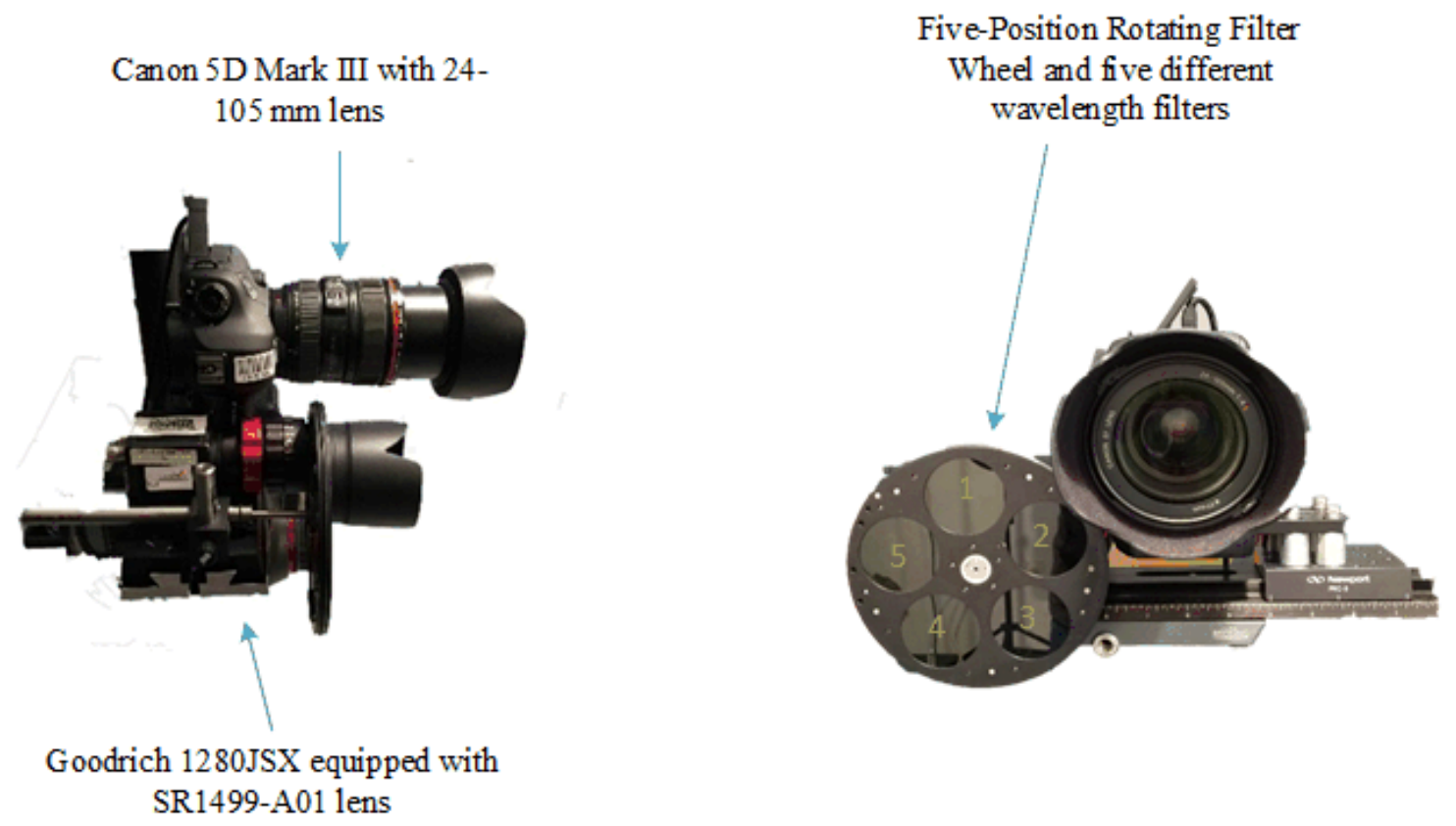

Figure 3.3: The collection device setup, Canon 5D Mark III, Goodrich IR camera and 5 position rotating filter wheel with 5 different wavelength filters.

frame rate, $1280 \times 1024$ pixel format and $1250 \mathrm{~nm}$ pitch. This camera offers the real-time daylight to low-light imaging via continuous monitoring laser detection in the SWIR wavelength spectrum, easily penetrates through dust and smoke. The high-dynamic-range helps camera to make a reliable imaging at nighttime without flashing and blooming. In this work, we equipped Stingray Optics Fuchsia SR1499-A01 lens with Goodrich SWIR camera to collect SWIR tattoo images.

- Five-Position Rotating Filter Wheel: The 5-position filter wheel with five filters of different wavelength bands is used to apply for different wavelength conditions. The Fig. 3.3 shows the structure of this filter wheel. The diameter of the wheel is 6 inches. Five band pass filters are placed on the each of holes in the wheel. The filters are used for this work are $1100 \mathrm{~nm}, 1200 \mathrm{~nm}, 1300 \mathrm{~nm}, 1400 \mathrm{~nm}$ and $1500 \mathrm{~nm}$. 


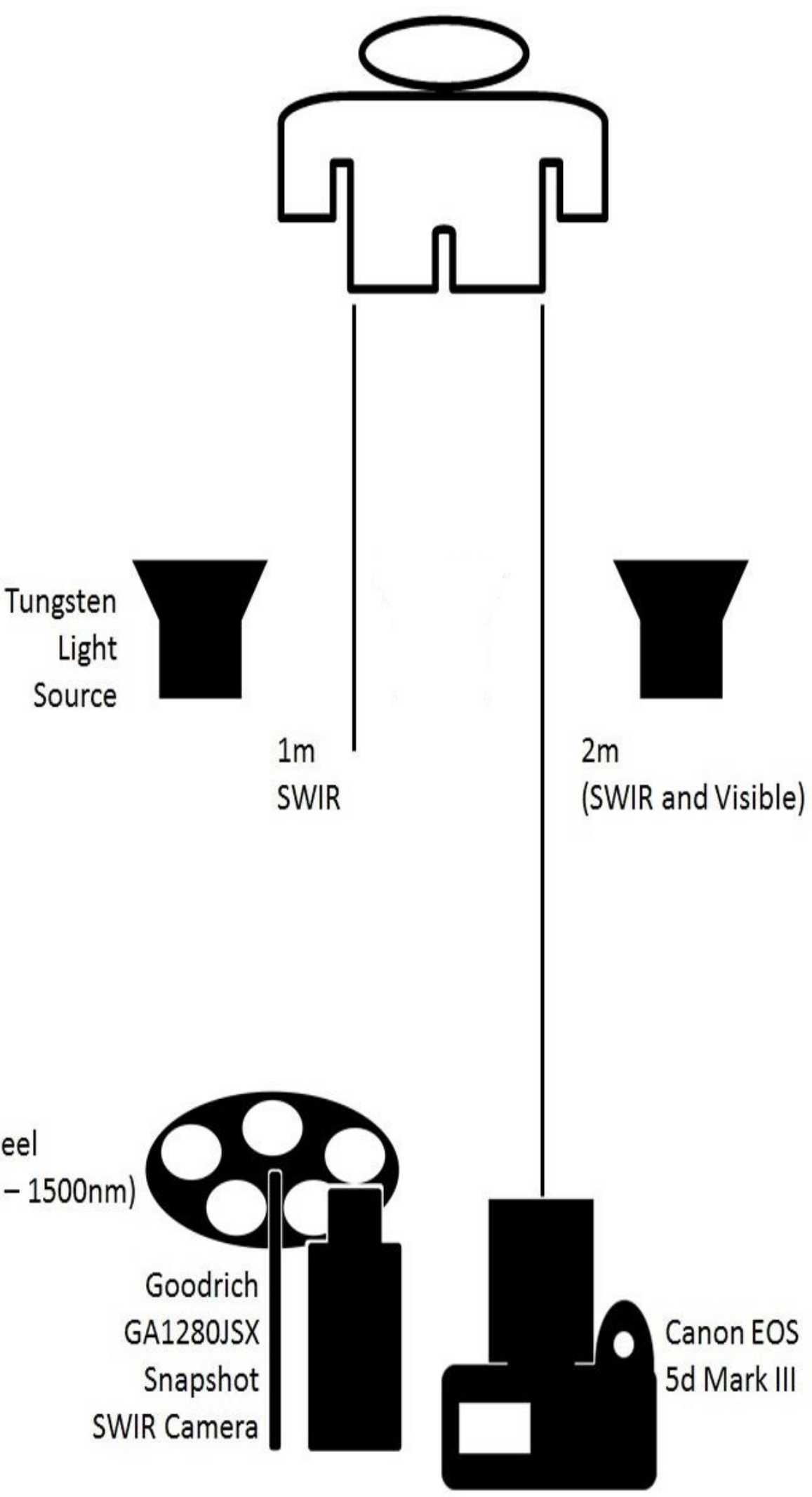

Figure 3.4: The overview of data collection setup for SWIR and visible bands tattoo image collection. 


\begin{tabular}{|l||c|c|c|c|}
\hline Database & Cameras & Num of Subject & Num of Session & Num of images \\
\hline \hline WVU-Tatt & Canon 5D Mark III & 96 & 1 & 5 \\
\hline
\end{tabular}

Table 3.1: The summary of each database setup for the experiments of cross-spectral matching.

\subsection{Experimental Setup}

\subsubsection{Cross-Spectral Tattoo Matching}

In the cross-spectral tattoo matching experiments, first of all, both $\underline{\mathrm{SWIR}}$ and visible bands of tattoo images were collected under controlled conditions. The overview of the data collection setup is shown in Fig. 3.4. In the data collection process, we set up two distances, $1 m$ and $2 m$. The SWIR tattoo database was collected at both $1 \mathrm{~m}$ and $2 \mathrm{~m}$ with five pass bands filters, $1100 \mathrm{~nm}, 1200$ $\mathrm{nm}, 1300 \mathrm{~nm}, 1400 \mathrm{~nm}$ and $1500 \mathrm{~nm}$. The visible band tattoo images were collected at only $2 \mathrm{~m}$. The datasets were captured with $70 \mathrm{~mm}$ lens zoom and also zoomed to fit the tattoos. Each subject per dataset includes 10 tattoo images. There are 150 subjects in total which have one session and 79 subjects which have two fully completed sessions collected with at least one month between sessions. In this part of work, we only investigated the cross-spectral and intra-spectral matching performance with 96 subjects from the same session. In each subject, we randomly selected 5 images from each dataset (visible zoomed fit, $1 \mathrm{~m}$ of each $1100 \mathrm{~nm}$ to $1500 \mathrm{~nm}$ )). The Table 3.1 summarizes the database setup for the cross-spectral matching experiments in Chapter 5 .

\subsubsection{Tattoo Image Registration}

In the tattoo image registration experiments, two tattoo databases are used. One database is well known as the NIST-Tatt-C database published by [42]. The NIST-Tatt-C database provides five cases of data categories of tattoo databases to focus but not limited on tattoo similarity, tattoo identification, region of interest, mixed media and tattoo detection. In this thesis, we only used the category of tattoo identification to evaluate this two-level based tattoo registration system. In tattoo identification category of NIST-Tatt-C database, we used 109 subjects to test our system. Each subject has one probe and one reference tattoo image that collected under uncontrolled condition. 


\begin{tabular}{|l||c|c|c|}
\hline Database & Cameras & Num of Subject & Num of Session \\
\hline \hline NIST-Tatt-C & Unknown & 109 & 2 \\
\hline WVU-Tatt & Canon 5D Mark III & 79 & 2 \\
\hline
\end{tabular}

Table 3.2: The summary of each database setup for the experiments of tattoo registration

Another database is the WVU-Tatt, which has been introduced in Section 3.2.1, the 79 subjects have been collected under controlled conditions with two sessions, and only visible collection has been used in this work. We randomly selected 1 of 10 images from each session per subject. The Table 3.2 summarizes the database setup for the experiments of tattoo image registration in Chapter 5. 


\section{Chapter 4}

\section{Methodological Approach}

In this chapter, we discuss our methodological approach to demonstrate that how we manage to efficiently match multi-wavelength (probe set) tattoo images, captured under different five SWIR filters applied against their tattoo counterparts (gallery set) captured under non-filter, visible condition. The overall process is illustrated in Fig. 4.1. Also this chapter provides the detailed approach of the automatic tattoo registration system.

\subsection{Cross-spectral Tattoo Matching}

\subsubsection{Geometric Normalization}

As the pre-processing step of cross-spectral tattoo image matching, we aligned the probe tattoo image (each SWIR band of tattoo datasets) to the reference one (visible tattoo dataset) by applying image rotation and scaling manually. The general process is shown in Fig. 4.1. In this part, we need to select two points from both of probe and reference images manually to make sure these two pairs of points coming from the similar tattoo pattern location as far as possible. Lastly, all processed tattoo images are downscaled to the same resolution. In this work, the resolution we used is $128 \times 128$ pixels. 


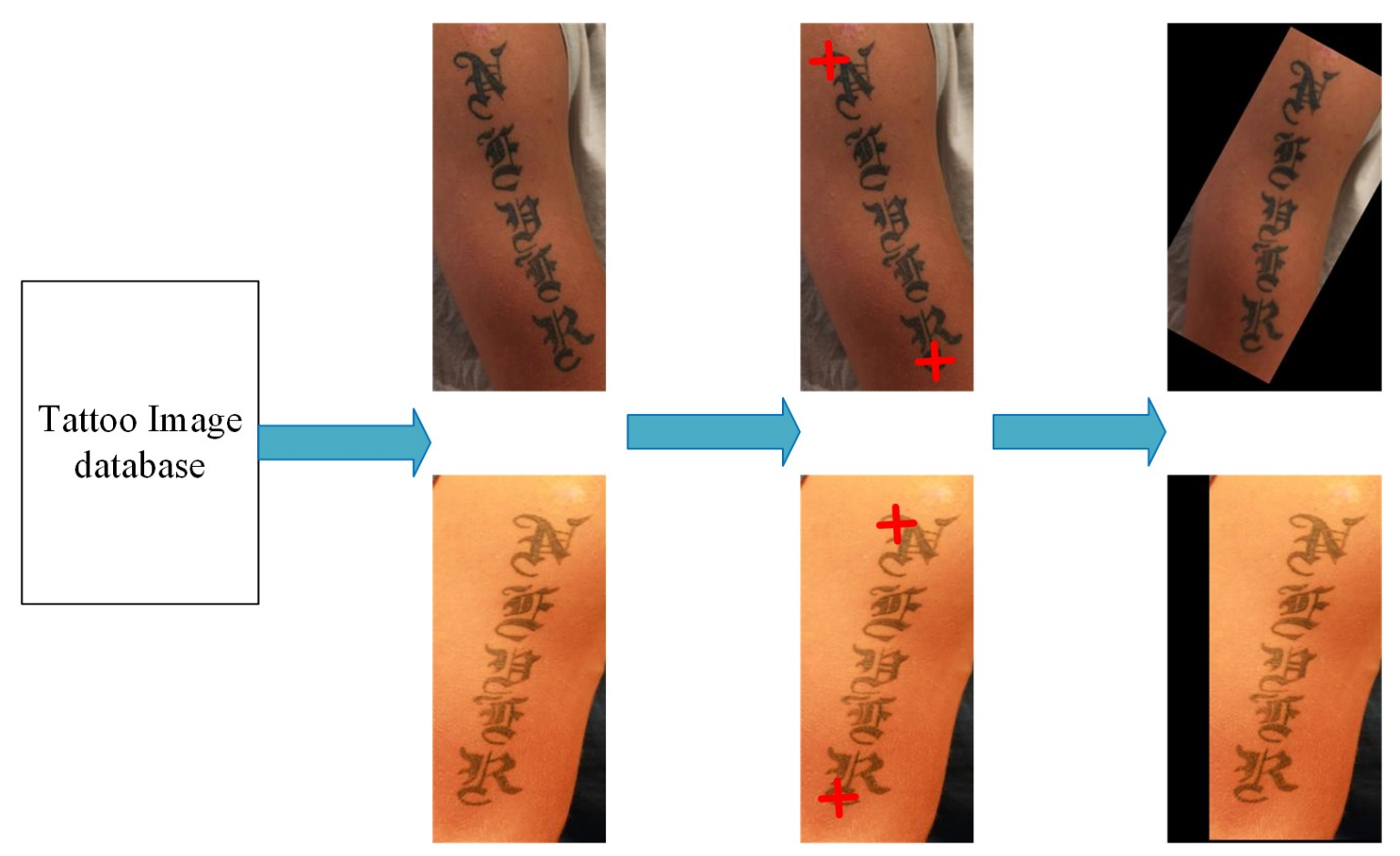

Figure 4.1: Overview of the tattoo rotation and cropping process.

\subsubsection{Photometric Normalization}

Illumination Normalization technique or as known photometric normalization is a very effective technology to achieve a positive influence to the human recognition systems. The photometric normalization aims to solve and reduce the influence of variable illumination like the reflect of light, dark light and different wavelength light. For this work, because the light source is controllable, we apply these techniques to alleviate the influence of SWIR and visible band tattoo images, and robust the local features of the raw tattoo datasets.

In our work, we tested twenty-one different photometric normalization techniques in total, twenty algorithms are from INface (Illumination Normalization techniques for robust Face Recognition) toolbox v2.0 [57] [58], to select the one which has the best performance for our Multiwavelength tattoo database. The following contents provide more specific details for each photometric normalization technique we tested:

- Single Scale Retinex (SSR): Jobson et al. [59] proposed the SSR algorithm which applied the majority of photometric normalization technique based on the retinex theory [60] and used the Gaussian filter for normalization. 
- Adaptive Single Scale Retinex (ASR): Park et al. [61] proposed the ASR method which is another additional approach based on retinex technique.

- Homomorphic Filtering based Normalization (WOMO): Heusch et al. introduced the HOMO method which is one of the widely used photometric normalization approach. This method can enhance the high frequency components of the input image also reduce the low frequency components by implementing Fourier transform and then by exploiting the inverse Fourier transform to transfer the input image from the frequency domain to spatial domain.

- Single Scale Self Quotinet (SSQ): Wang et al. [62] proposed the SSQ algorithm which is very similar to the $\mathrm{SSR}$ algorithm. Unlike SSR using a Gaussian filter, the SSQ uses Gaussian smoothing filter.

- Multi Scale Self Quotinet (MSQ): Wang et al. [62] introduced another photometric normalization technique in their work called MSQ which is still based on Gaussian smoothing filter.

- Discrete Cosine Transform based Normalization (DCT): Chen et al. [63] proposed the DCT based normalization technique. It mainly operates an input image into the low frequency domain by calculating a number of DCT coefficients and minimizing variations to attain illumination invariance.

- Wavelet based Normalization (WA): Du and Ward [64] introduced the WA method which is based on implementing the discrete wavelet transform.

- Wavelet Denoising based Normalization (WD): Zhang et al. [65] developed the WD technique which is based on implementing the wavelet denoising.

- Isotropic Diffusion based Normalization (IS): Heusch et al. [66] introduced the IS]normalization method which is based on the anisotropic diffusion. It applies an isotropic smoothing function for the input image to acquire the information of luminance.

- Anisotropic Diffusion based Normalization ( $\underline{\mathbf{A S}}$ ): Gross and Brajovic [67] proposed the AS algorithm by implementing the normal anisotropic smoothing to the input image to evaluate the luminance information. 
- Steerable Filter based Normalization $(\underline{\mathrm{SF}})$ : The $\underline{\mathrm{SF}}$ is an effective approach to remove the variations caused by illumination from the original images through steerable filters.

- Non-local Means based Normalization (LM): Štruc and Pavešić [68] proposed the NLM technique which is based on the non-local means denoising method to evaluated the reflectance and luminance information.

- Adaptive Non-local Means based Normalization (ANL): Štruc and Pavešić [68] proposed another photometric normalization technique called ANL which introduced an adaptive way to use non-local means denoising algorithm to obtian the reflectance and the luminance function.

- Modified Anisotropic Diffusion Normalization (MAS]: Gross and Brajovic [69] introduced the MAS method which is based on AS and includes the post-processing procedure.

- Gradientfaces Normalization (GF): Zhang et al. [70] proposed the GF algorithm which is based on the computation results of the image gradients and combining with the orientation information to yield an image with illumination invariant.

- Single Scale Weberfaces Normalization (SSW): Wang et al. [71] introduced the SSW approach which is based on Weber contrast and image gradients to represent an illumination invariant image.

- Multi scale Weberfaces normalization (MSW): Wang et al. [71] also proposed an extension work of $\mathrm{SSW}$ called $\mathrm{MSW}$ which is not only to combine Weber contrast and image gradients but also implement a linear combination to represent the illumination invariant image.

- Large- and small-scale Features Normalization ([LSSF): Xie et al. [72] introduced the LSSF method by using both large and small scale features as the principal components to process the input image. The reflectance and luminance functions are calculated as the first round, and then the same process is applied as the second round of normalization to finally get output image. 
- Tan and Triggs Normalization (TT): Tan and Triggs in [73] proposed the TT normalization which is based on gamma correction, DoG filtering and post-processing step to represent the output image.

- DoG Filtering based Normalization (DoG): The DoG technique is based on applying the difference of Gaussian filter to construct the normalized image. Either gamma correction or logarithm transform is required to process input image first according to INFace toolbox [57] [58].

- CLAHE: CLAHE is a very popular normalization technique widely used in the field of face recognition proposed in [25] by Zuiderveld. It separates the input image to several small patches which are called tiles and enhances each of patches individually.

Finally, TT photometric normalization has been selected to operate the rest of cross-spectral matching experiments based on the reliable performance and the best results for matching bands of $1100 \mathrm{~nm}, 1200 \mathrm{~nm}$ and $1500 \mathrm{~nm}$ against to visible set (see Chapter 5 and Fig. 5.3, Fig. 5.4, Fig. 5.5, Fig. 5.6, Fig. 5.7 for more details). The Fig. 4.2 exhibits the processed results of all photometric normalization techniques, the input image is captured in visible band. Fig. 4.3 shows an example of the processed tattoo images in all bands (visible vs. SWIR, original vs. TT).

\subsubsection{Feature Extraction and Matching}

In this section, two main matching methods we used in this work are introduced, SIFT and LBP They are both very popular and successful approaches applied for face and object recognition.

\section{Local Binary Patterns}

The LBP operator was first proposed by Ojala et al. [74] and it is one of the best widely used algorithms for texture features representation and description in various component-based human recognitions.

The original operator of LBP sets the entire pixels of the input image through thresholding the $3 \times 3$ neighborhood of each pixel with the central pixel value and generating 8-bit binary array as the result. Then, a texture descriptor is created through the histogram of the labels. For instance, 


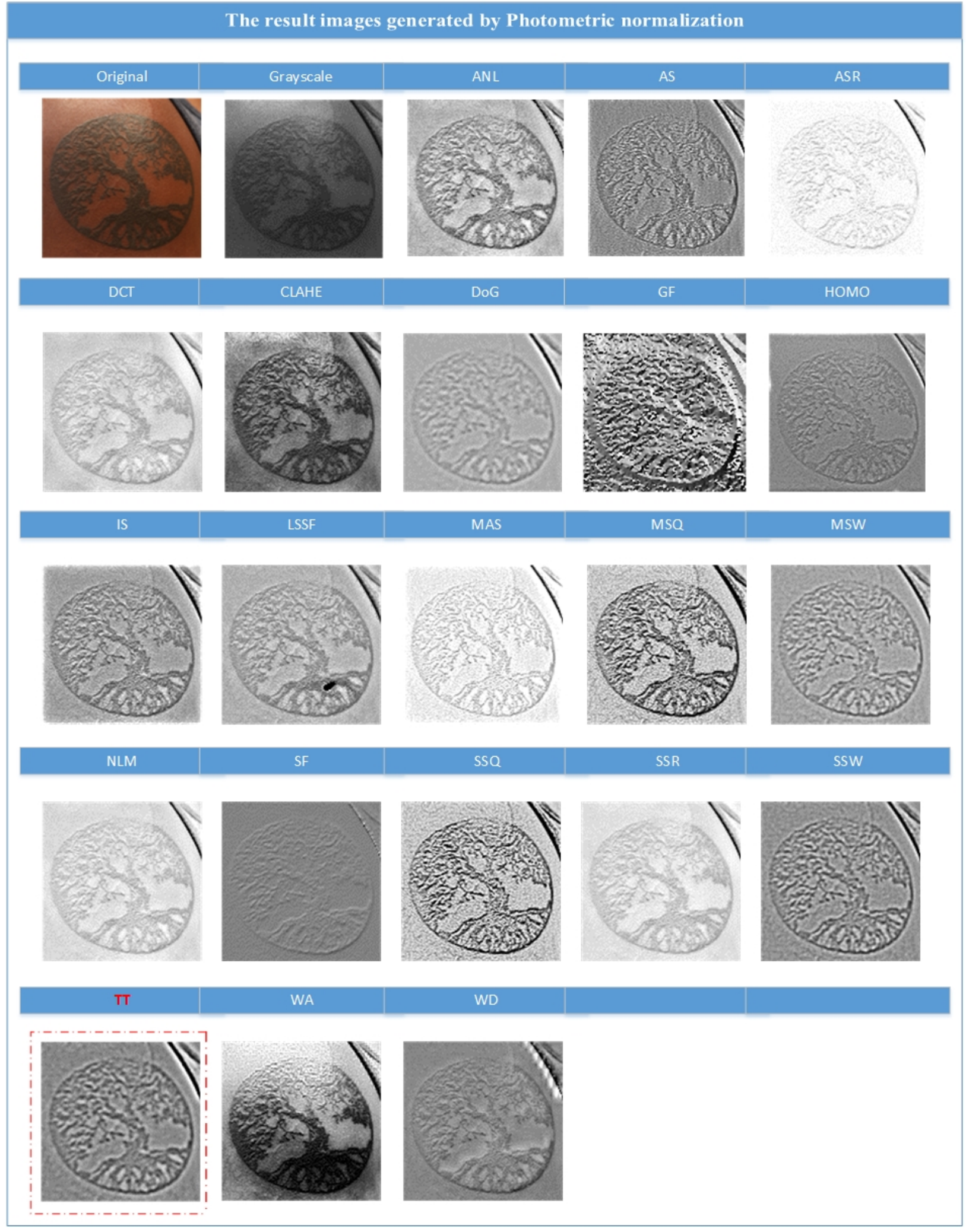

Figure 4.2: The results of photometric normalization. The red box shows the Tan and Triggs result which we selected for our entire set of experiments. 

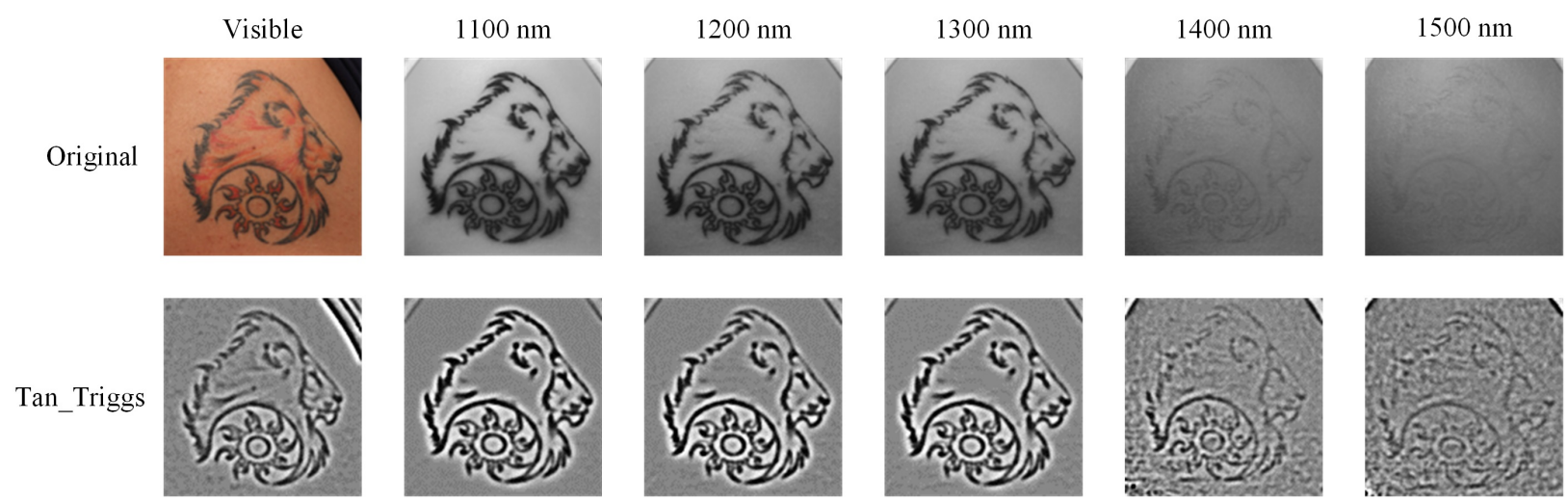

Figure 4.3: The results of one of the photometric normalization methods, TT method is applied for all bands of SWIR and visible tattoo image

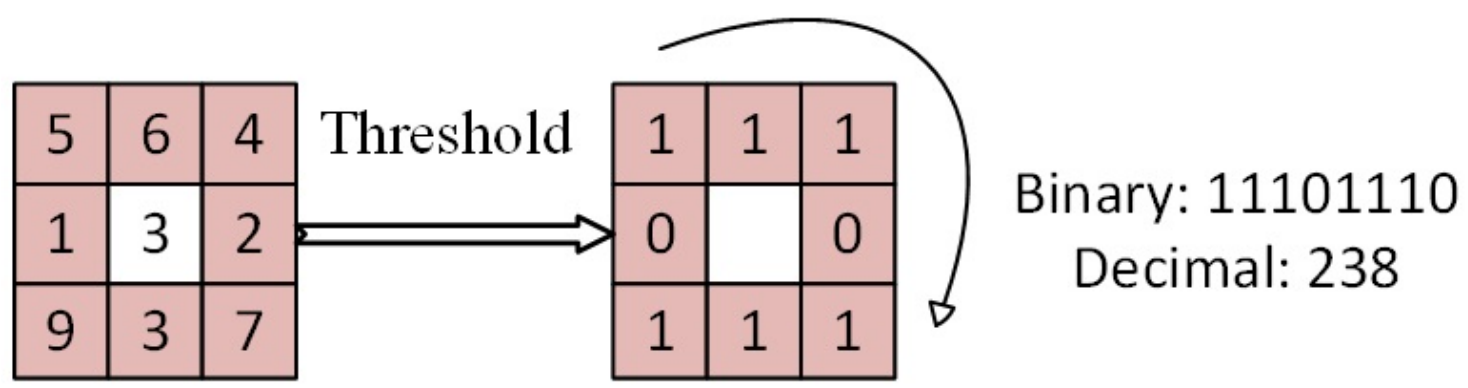

Figure 4.4: A demonstration of how a basic LBP operator works.

Fig. 4.4 shows the sample process of $\mathrm{LBP}$ operator in 9 pixels consisting with a $3 \times 3$ block. The central pixel value is 3 . By comparing the value of all 8 neighbor pixels with the central value, all the pixels value greater or equal to 3 will be recorded as 1 in the new $3 \times 3$ block at the same position, otherwise, the pixels will be set to 0 . Therefore, a new block figure can be obtained (See the right side block of Fig. 4.4. Then an 8-bit strings as a binary array can be gained, the value is 11101110 . Finally, the central pixel of this block can be represented by converting this binary array to decimal value. In this case, the decimal value is 238 .

The original LBP operator has been proved as a powerful texture measurement approach in many areas. But one of the disadvantage of this algorithm is that it is difficult to describe the information with different scales. To solve this issue, Ojala et al. [75] proposed an extensional version of LBP operator. The new LBP method, also referred as circular LBP, defines that the local neighborhood points are distributed evenly around the circle center. Through this way, the 


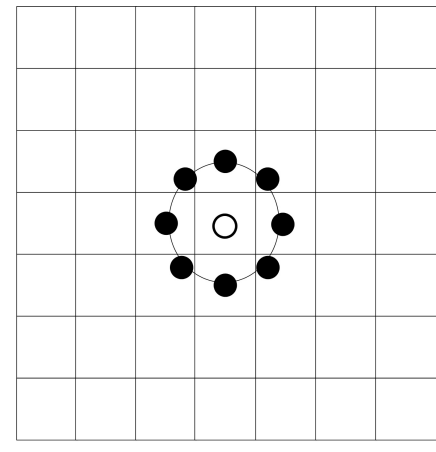

$\mathrm{P}=8, \mathrm{R}=1$

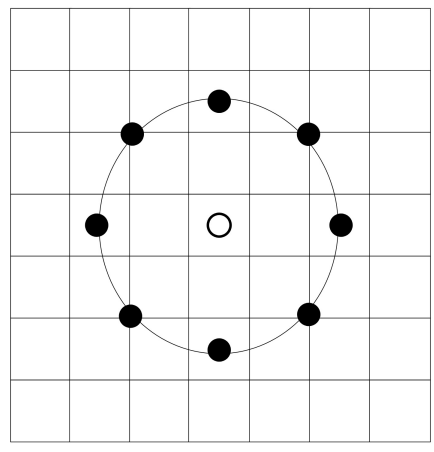

$\mathrm{P}=8, \mathrm{R}=2$

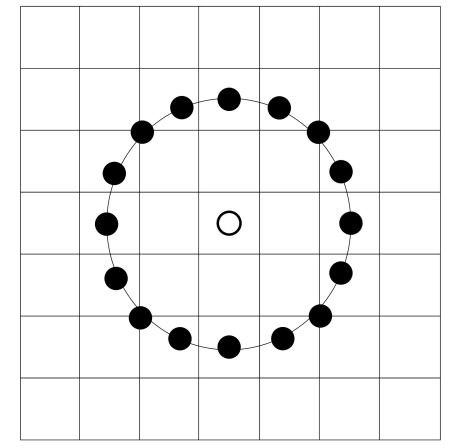

$\mathrm{P}=16, \mathrm{R}=2$

Figure 4.5: The example of circular LBP operator, the neighborhood points and radius are different, such as $(8,1),(8,2)$ and $(16,2)$, by bilinear interpolated.

radius and the number of points of LBP descriptors can be set to any available value according to the feature extraction purpose. If the sampling point cannot locate at the center of a pixel, the bilinear interpolation will be implemented. Here, the number of pixel neighborhoods is expressed as $P$ and the circle radius is defined as $R$. The example of circular LBP operator is demonstrated with different values of $P$ and $R$ in Fig. 4.5.

Another extension of LBP operator which played an important role in our experiments is uniform patterns proposed in [75]. The uniform pattern is defined only if a circular LBP descriptor includes no more than twice of bitwise transitions from 0 to 1 or 1 to 0 . For instance, the binary arrays 00000000 (0 transition) and 00000001 (1 transition, from 0 to 1 ) can be defined as uniform patterns. The binary array 10000100 (3 transitions from 1-0-1-0, more than twice) cannot be defined as uniform pattern.

The main purpose to define the uniform patterns for $\mathrm{LBP}$ is to group all of the nonuniform patterns into a single bin and group each of uniform patterns into a different bin through the LBP histogram computation. There are existing experiments with texture images finished by Ojala et al. [75] and the results indicate that almost $90 \%$ of all patterns are the uniform patterns when using 8 neighborhood pixels and 1 as the radius $(\mathrm{P}=8, \mathrm{R}=1)$, about $70 \%$ of patterns are uniform patterns in the neighborhood of 16 and the radius as $2(16,2)$. Also in the work of Ahonen et al. [21], they found that, in FERET facial image database, $90.6 \%$ of the patterns are uniform patterns when using $(8,1)$ and $85.2 \%$ in the neighborhood of $(8,2)$.

In this work, the $\mathrm{LBP}$ operator is defined as $L B P_{P, R}^{u 2}$. The subscript expresses to set the neigh- 
borhood parameters in the LBP operator $(P, R)$, the value of $\mathrm{P}$ stands for number of neighbor pixels used and $\mathrm{R}$ means the radius set for the operator. The superscript $u 2$ indicates that only the uniform patterns are considered in this LBP descriptor. In general, we use an input pixel defined as $\left(x_{a}, y_{a}\right)$ through the LBP operator to get the resulting decimal LBP value by computing the following equation [76]:

$$
\operatorname{LBP}_{(P, R)}\left(x_{a}, y_{a}\right)=\sum_{p=0}^{P-1} S\left(g_{p}-g_{c}\right) 2^{P}
$$

where the $g_{p}$ and $g_{c}$ (i.e., $p=0,1, \ldots, P-1$ ) stand for the value of the central pixel and the $P$ and $R$ are the same as the notion $(P, R)$ which have been defined earlier. $2^{P}$ indicates a binomial weight assigned to each $S\left(g_{p}-g_{c}\right)$. The function $S(x)$ which associates to select uniform patterns can be expressed as follow:

$$
S(x)= \begin{cases}1, & x \geq 0 \\ 0, & x<0\end{cases}
$$

In our experiments, the parameters set of the LBP algorithm is from the work of Ahonen et al. [77]. We select the uniform patterns from LBP descriptors with $18 \times 21$ pixel windows and $P=8, R=2$. This operator can be expressed as $L B P_{8,2}^{u 2}$ since Ahonen et al. verified that this is the best parameter setup to get the best matching accuracy.

\section{Scale-Invariant Feature Transform}

According to [20], the SIFT feature is invariant to translation, rotation and scale. It is a kind of very robust local feature descriptor that has been applied for many research areas, such as object detection, tracking, recognition, tattoo retrieval, and matching.

The main four steps described below are the general procedure of the SIFT features computation:

- Scale-space extrema detection

- Keypoint localization

- Orientation assignment

- Keypoint descriptor 
The general methodological approach can be described as follow equations:

$$
\begin{aligned}
D(x, y, \sigma) & =(G(x, y, k \sigma)-G(x, y, \sigma)) * I(x, y) \\
& =L(x, y, k \sigma)-L(x, y, \sigma)
\end{aligned}
$$

where $k$ is defined as a constant multiplicative factor, and $L(x, y, \sigma)$ is the scale space function can be expressed as

$$
L(x, y, \sigma)=G(x, y, \sigma) * I(x, y)
$$

where $G(x, y, \sigma)$ is the convoluted variable-scale Gaussian shown as

$$
G(x, y, \sigma)=\frac{1}{2 \pi \sigma^{2}} e^{-\left(x^{2}+y^{2}\right) / 2 \sigma^{2}}
$$

$I(x, y)$ stands for an input image.

After extracting the SIFT features and descriptors from a probe and gallery image (defined as $D_{1}$ and $D_{2}$ ), the squared Euclidean distance Dist between $D_{1}$ to all $D_{2}$ can be calculated, the keypoints can match with each other only if the criteria of Eqn. 4.6 can be satisfied (see [20] and [78] for more details). In this work, the value of threshold is 1.5 which is the default value of the SIFT method provided by [78].

$$
\operatorname{Dist}\left(D_{1}, D_{2}, \text { threshold }\right)<\operatorname{Dist}\left(D_{1}, D_{2}\right)
$$

\section{Distance metrics}

In computer vision and biometrics, distance metrics are played an important role in many tasks like image classification, identification and image quality assessment. In general, the distance metrics are usually applied to calculate the distance between two feature vectors and feature descriptors of images.

\section{- Euclidean Distance:}

In our work, we used squared Euclidean Distance to measure two SIFT descriptors to calculate the final distance scores by applying the VLFeat toolbox [78]. The equation can be described below: 


$$
d^{2}=\sum_{i=1}^{n}\left(D_{1 i}-D_{2 i}\right)^{2}
$$

where $d^{2}$ is the squared Euclidean Distance, $D_{1}$ and $D_{2}$ stand for descriptor 1 and descriptor 2 of SIFT.

- Chi Squared Distance:

According to the work of Ahonen et al. in [77], the Chi-squared $\left(\chi^{2}\right)$ histogram distance is named as the distance measurement tool to calculate the distance between two feature vectors of $L B P_{8,2}^{u 2}$ descriptors. The equation below shows the expression of $\chi^{2}$ :

$$
\chi^{2}(p, q)=\sum_{i} \frac{\left(p_{i}-q_{i}\right)^{2}}{p_{i}+q_{i}}
$$

where $p$ and $q$ are the two histogram vectors of the $L B P_{8,2}^{u 2}$ descriptors. In our experiments, the Chi squared distance is also used as the distance metric for the LBP method.

\subsection{Automatic Tattoo Image Registration System}

In this section, we introduce our proposed automatic tattoo registration system detailed. Our developed system includes three main approaches: (A) tattoo image registration with SIFT descriptor and RANSAC algorithm; (B) registration evaluation and (C) registration error correction. The overall process is illustrated in Fig. 4.6 .

\subsubsection{Tattoo Image Registration with SIFT Descriptor and RANSAC Algo- rithm}

The methodological approach and the acquirement of SIFT descriptors has been described in the previous section.

The RANSAC algorithm is an effective approach to filter the outliers of SIFT matchers and improve the reliability of SIFT keypoint matching through a statistic way [22]. In the proposed tattoo registration system, we use the RANSAC algorithm associated with a homography model to 


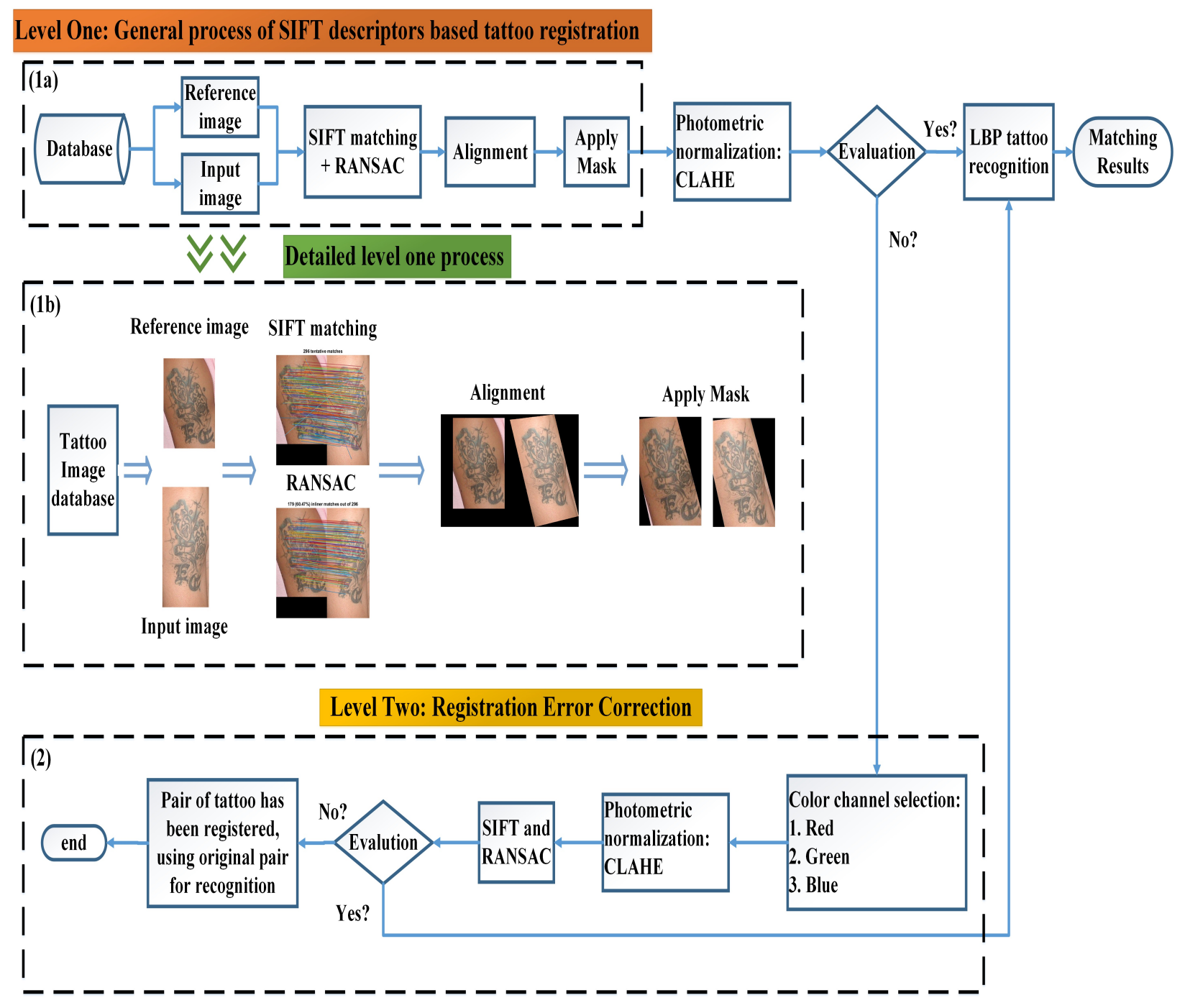

Figure 4.6: The overall approach flowchart of our proposed two-level based automatic tattoos registration system. Block (1b) indicates the detailed level one registration process of block (1a) applied SIFT and RANSAC with homography model. Block (2) shows the general registration process of level two (see Fig. 4.8 for more details about level two process). 
filter outliers of SIFT matcher and align the tattoo images to achieve the registration purpose [78] (see block (1b) of Fig. 4.6 for the process of SIFT and RANSAC).

\subsubsection{Registration Evaluation}

After getting the pair of registered tattoos by the registration system of level one, it is very important to check whether this pair of tattoos are aligned in the right way or not. The traditional way to finish this work is applying manual evaluation by a human operator. However, manual evaluation has major disadvantages when compared to an automated evaluation system. Firstly, it is unrealistic to adapt large dataset measurement by human operators because a long time will be taken if we visually judge the registration results. Secondly, visual measurement cannot guarantee the accuracy of registration results because our eyes are not accurate enough to distinguish small difference between two similar images. To deal with these two problems, we design a scheme to check and judge the validity of registration results automatically.

In our designed method, we computer and compare the similarity scores between original pair and registered pair of tattoo images which have been applied CLAHE technique first, then fuse the two image quality index methods, UIQ and FSIM, in the score level. The fundamental principle of this approach is demonstrated in Fig. 4.7. The process of score level fusion can be described as follow: 1) normalize scores of UIQ and FSIM from 0 to 1 which means that if the score is more closed to 1 , the pair of tattoo images is more similar with each other, and vice versa; 2) sum the normalized UIQ and FSIM scores to get a new fusion score that used to judge and decide the validity of registration. The original fusion score is defined as Score1, which can be computed by using the original pair of tattoo images that applied the score fusion scheme, the registered fusion score is defined as Score2, which can be obtained by calculating the registered pair of tattoo images using this score fusion scheme. The difference value (Dval) between Score 1 and Score 2 is implemented to decide whether this pair of tattoo images is registered successfully or not (regard Eqn. 4.9). If Dval is greater than zero, it will indicate that the registration is successful. If not, it will indicate that the registration is failed. 


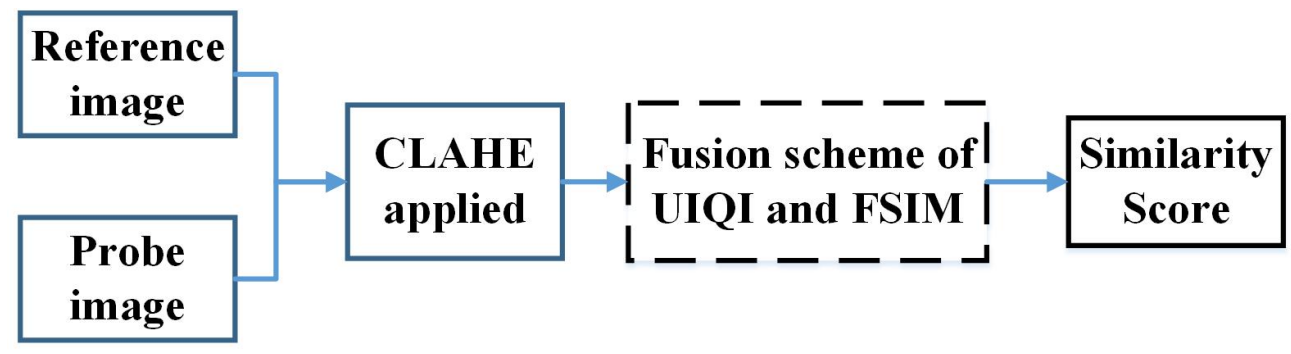

Figure 4.7: The general process of score fusion based image quality index technique.

$$
\text { Dval }=\text { Score } 2-\text { Score } 1 \begin{cases}>0, & \text { correct } \\ <0, & \text { incorrect }\end{cases}
$$

\subsubsection{Registration Error Correction}

Although SIFT descriptors offer robust local features and RANSAC can filter outliers of SIFT matching keypoints, the tattoo registration results still suffer from mismatch and lack of SIFT keypoints matched in several subjects. In these specific cases, several adverse factors may be considered, such as distortion, illumination and low quality, which may significantly reduce the robustness of local SIFT descriptors. In order to overcome these challenges, the R, G, B color representation of the input image and CLAHE method [25] are combined and applied as the main approach of second level of our registration system. The second level of this registration system aims to enhance local SIFT features and reduce the impact of unfavorable factors. Fig. 4.8 demonstrates the detailed process of registration error correction.

To find out which color channel-CLAHE integration method has the best performance in this work, we analyzed all the SIFT keypoint matching for each color channel combined with CLAHE technique for the subjects which cannot be registered correctly by system of level one. Fig. 4.9 shows an example of a comparative study for an subject which cannot be registered successfully, (A) demonstrates the level one SIFT descriptors match, there are only 5 keypoints can be matched with each other and several mismatch keypoints still existing. (B), (C) and (D) indicate red channel, green channel and blue channel with CLAHE technique applied for the registration error correction. The red channel with CLAHE technique has the best performance in both number of keypoint 


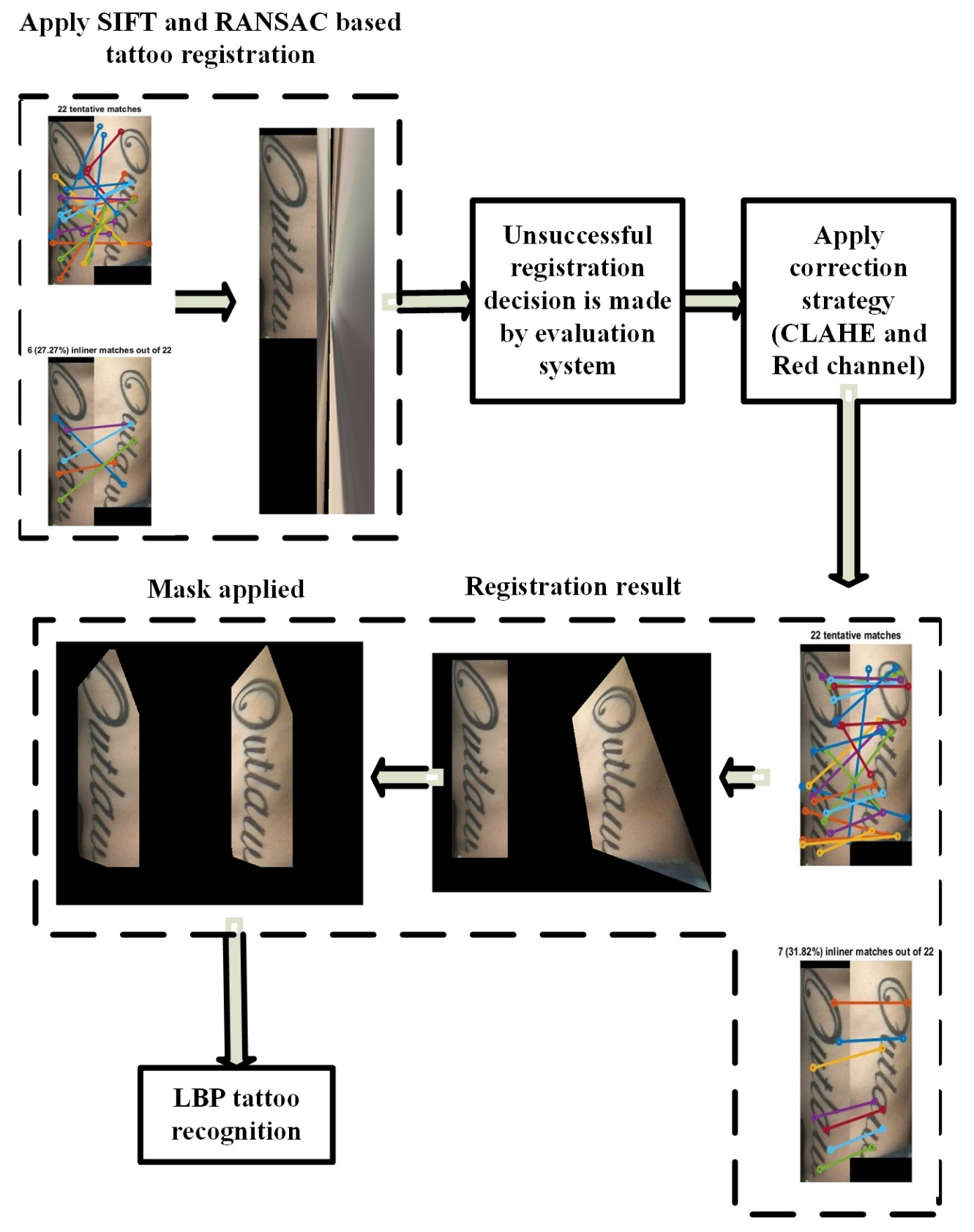

Figure 4.8: The general process of second level based tattoo registration system. The upper block shows an example of incorrect registration makes by level one of our system. The lower block indicates the result of level two registration after applying red-channel-CLAHE for the input pair tattoos. 
SIFT matching with RANSAC on grayscale tattoo image

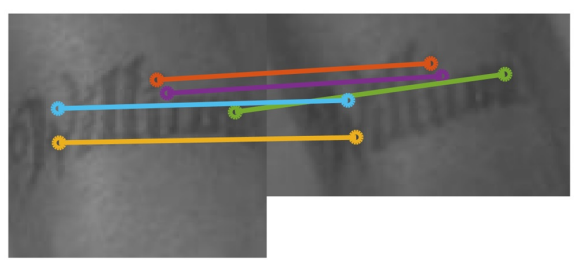

(A)

SIFT matching with RANSAC on greenchannel tattoo image applied CLAHE

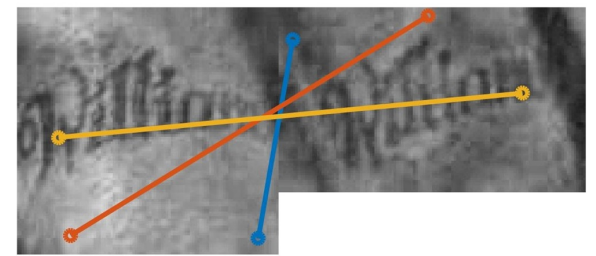

(C)
SIFT matching with RANSAC on redchannel tattoo image applied CLAHE

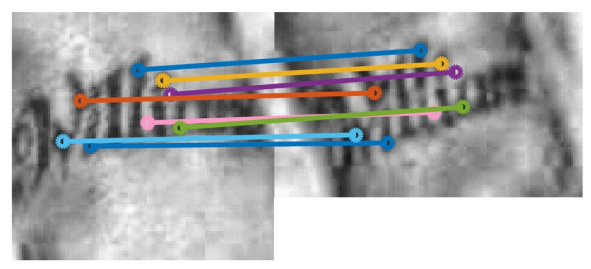

(B)

SIFT matching with RANSAC on bluechannel tattoo image applied CLAHE

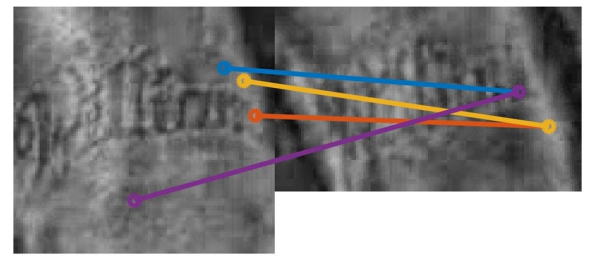

(D)

Figure 4.9: Tattoo Image analysis of SIFT matching for all grayscale (A), red-channel CLAHE (B), green-channel CLAHE (C) and blue-channel CHALHE (D).

matched and number of keypoint matched correctly. Table 4.1 summarized all the unsuccessfully registered subjects for both databases we used to test this registration system. From the results shown in Table 4.1, the red channel with CLAHE combination is always the best one for all incorrectly registered subjects. Therefore, we choose the red channel integrated with CLAHE technique for the registration error correction process. 


\begin{tabular}{|l||c|c|c|c|}
\hline Subject & Grayscale & R-CLAHE & G-CLAHE & B-CLAHE \\
\hline \hline NIST-Tatt-C 1 & $4(2)$ & $\mathbf{7 ( 7 )}$ & $4(2)$ & $0(0)$ \\
\hline NIST-Tatt-C 2 & $6(2)$ & $\mathbf{7 ( 7 )}$ & $8(4)$ & $16(3)$ \\
\hline NIST-Tatt-C 3 & $4(2)$ & $\mathbf{8 ( 8 )}$ & $4(0)$ & $4(0)$ \\
\hline NIST-Tatt-C 4 & $5(1)$ & $\mathbf{8 ( 8 )}$ & $7(0)$ & $5(0)$ \\
\hline NIST-Tatt-C 5 & $5(4)$ & $\mathbf{8 ( 8 )}$ & $4(0)$ & $4(0)$ \\
\hline NIST-Tatt-C 6 & $4(0)$ & $\mathbf{1 1}(\mathbf{1 0})$ & $5(2)$ & $6(2)$ \\
\hline WVU-Tatt 1 & $7(1)$ & $\mathbf{2 6 ( 2 6 )}$ & $5(0)$ & $9(1)$ \\
\hline WVU-Tatt 2 & $7(6)$ & $\mathbf{8 ( 8 )}$ & $6(3)$ & $7(1)$ \\
\hline WVU-Tatt 3 & $4(1)$ & $\mathbf{1 2}(\mathbf{1 2})$ & $4(1)$ & $3(1)$ \\
\hline
\end{tabular}

Table 4.1: Summary of R, G, B channel combined with CLAHE for all the incorrectly registered subjects for both databases (NIST-Tattoo-C and WVU-Tatt). Numbers outside the parenthesis represent the number of SIFT keypoint matching, numbers inside the parenthesis represent the correct SIFT keypoint matching. 


\section{Chapter 5}

\section{Experimental Results of Tattoo Matching and Registration}

This chapter describes and summarizes all the experimental results of cross-spectral tattoo recognition and tattoo registration system including: (1) descriptor selection between $\mathrm{LBP}$ and SIFT, (2) photometric normalization method selection, (3) tattoo cross-spectral matching with or without photometric normalization applied, intra-spectral tattoo matching with photometric normalization, (4) results of tattoo registration in visible band. The first three sections are focus on the first problem: cross-spectral matching and the section 4 is focus on exploring tattoo registration approach in visible band only.

\subsection{SIFT vs. LBP Descriptors}

The experiment of this section aims to explore which descriptor, SIFT or LBP, is better to implement for cross-spectral tattoo matching. In this section, all the tattoo images are original cropped and resized to $128 \times 128$ without any photometric normalization techniques applied. Fig. 5.1 indicates the Cumulative Match Characteristic (CMC) curves of LBP with uniform patterns associated with Chi square distance measurement and the table below the CMC curves shows the accuracy of rank-1 to rank-5 of matching results. Fig. 5.2 indicates the CMC curves of SIFT method associated with Euclidean distance measurement and also the table below the curves represents the accuracy of rank-1 to rank- 5 of matching results. By comparing these two figures, we can 


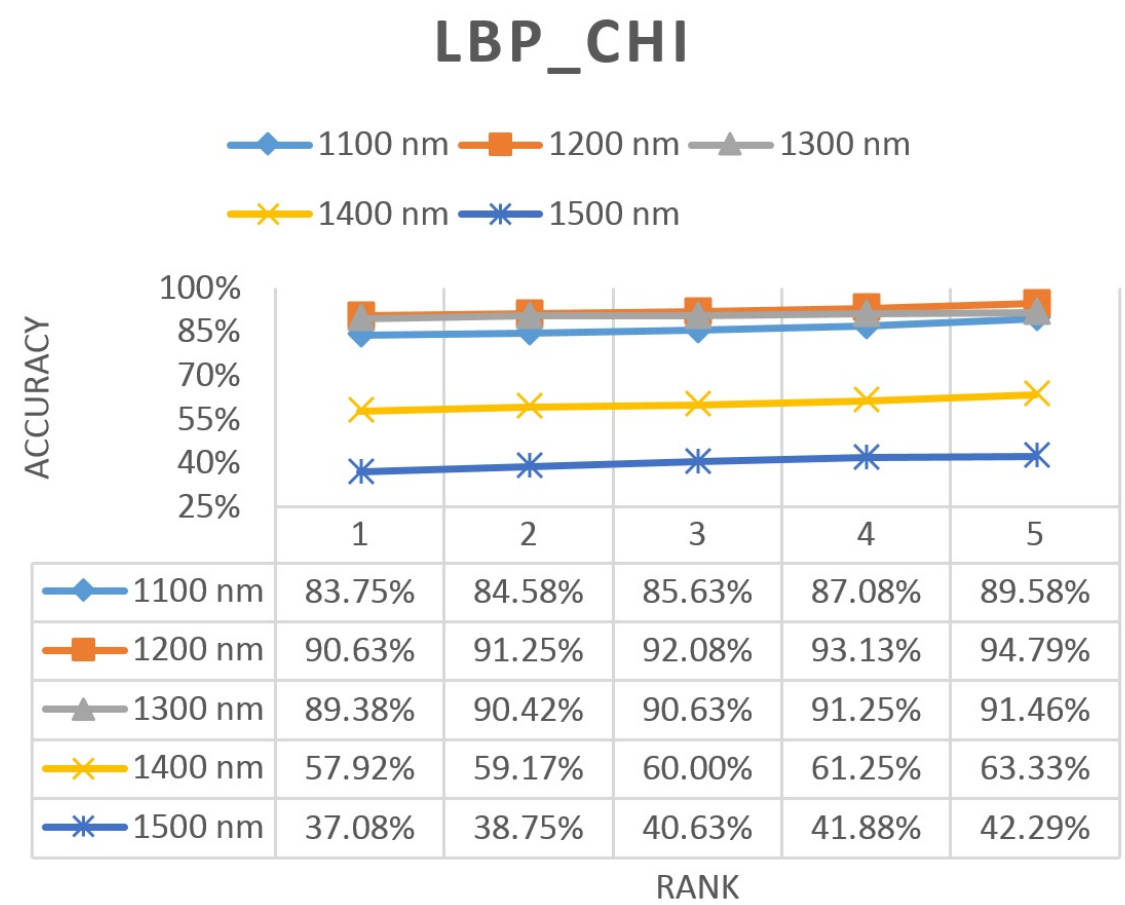

Figure 5.1: The CMC curve of cross-spectral tattoo matching applied for LBP method measured by Chi squared.

easily conclude that LBP descriptor is much better than SIFT descriptor for cross-spectral tattoo image matching. The accuracy for each band has been significantly improved by LBP method. The performance of SIFT method is very poor, especially for $1400 \mathrm{~nm}(9.79 \%)$ and $1500 \mathrm{~nm}(3.13 \%)$. Therefore, SIFT descriptor is not useful for cross-spectral tattoo image matching. We will use LBP descriptor for further experiments.

\subsection{Exploring Photometric Normalization Techniques}

The purpose of this section is to explore and solve one question: Can photometric normalization techniques help us to improve the matching accuracy for cross-spectral tattoo matching? To deal with this problem, first of all, all the tattoo images (both of visible and SWIR images) are processed by 21 photometric normalization techniques separately. Then, we have done a comparative matching for each photometric normalization method for each SWIR band by extracting LBP features. Fig. 5.3 shows the accuracies of rank-1 result for $1100 \mathrm{~nm}$ of each photometric 


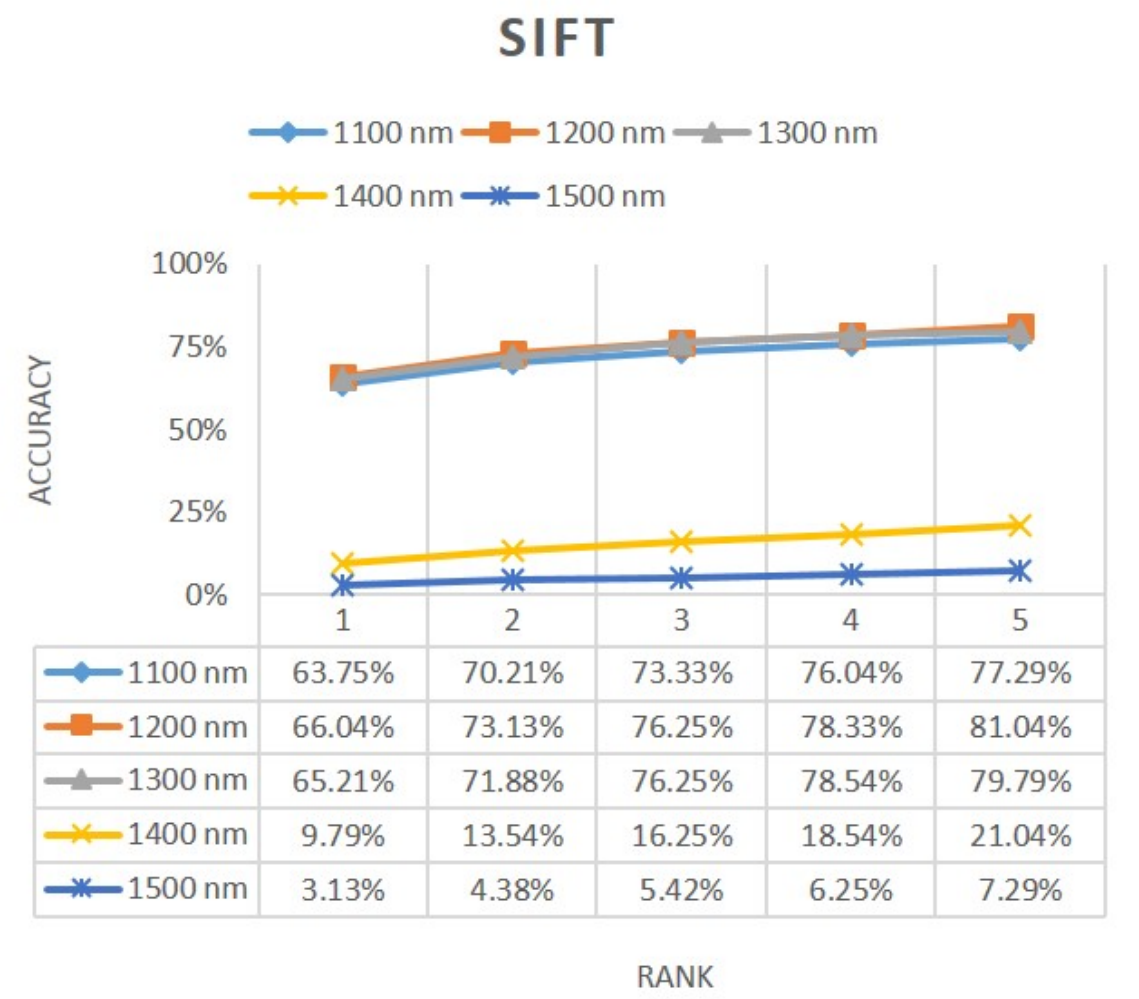

Figure 5.2: The CMC curve of cross-spectral tattoo matching applied for SIFT method measured by Euclidean distance.

normalization technique. Fig. 5.4 indicates the accuracies of rank-1 result for $1200 \mathrm{~nm}$ for each photometric normalization method. Fig. 5.5 exhibits the accuracies of rank-1 result for $1300 \mathrm{~nm}$. Fig. 5.6 shows the accuracies of rank-1 result for each photometric normalized technique for 1400 $\mathrm{nm}$ and Fig. 5.7 demonstrates the accuracies of rank-1 result for $1500 \mathrm{~nm}$.

From the results above, we can find out that the two photometric normalization techniques, DoG and TT, always demonstrate the best rank-1 results for each band among all 21 photometric normalization methods. For the bands of $1100 \mathrm{~nm}, 1200 \mathrm{~nm}$ and $1500 \mathrm{~nm}$, TT shows its advance and for $1300 \mathrm{~nm}$ and $1400 \mathrm{~nm}$, DoG method shows better matching accuracies. Fig. 5.8 and Fig.5.9 indicate the CMC curves for both TT and DoG methods. From these results, we can easily compare the accuracy of each method, band and rank (rank-1 to rank-5). By analyzing and comparing all the results of each band each method, we selected TT as the photometric normalization technique for the rest of experiments. Table 5.1 refers all rank-1 results for each band by applying TT and DoG or not. Row 1 shows the original result (no photometric normalization), row 2 indicates the 


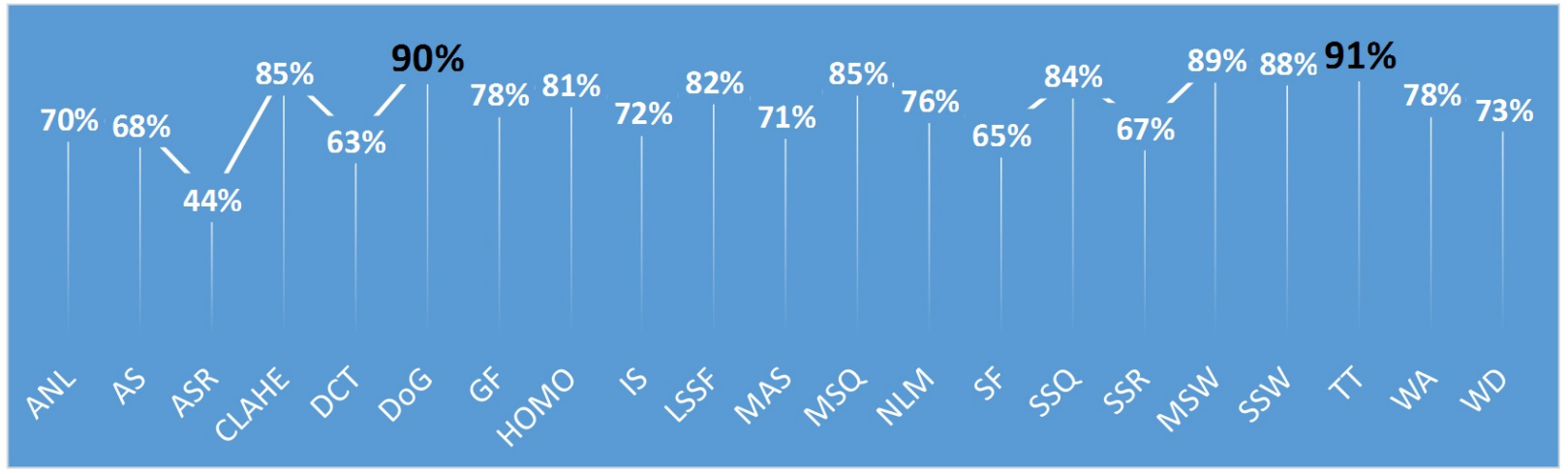

Figure 5.3: The results for $1100 \mathrm{~nm}$ vs. gallery tattoo matching applied for each of the 21 photometric normalization techniques.

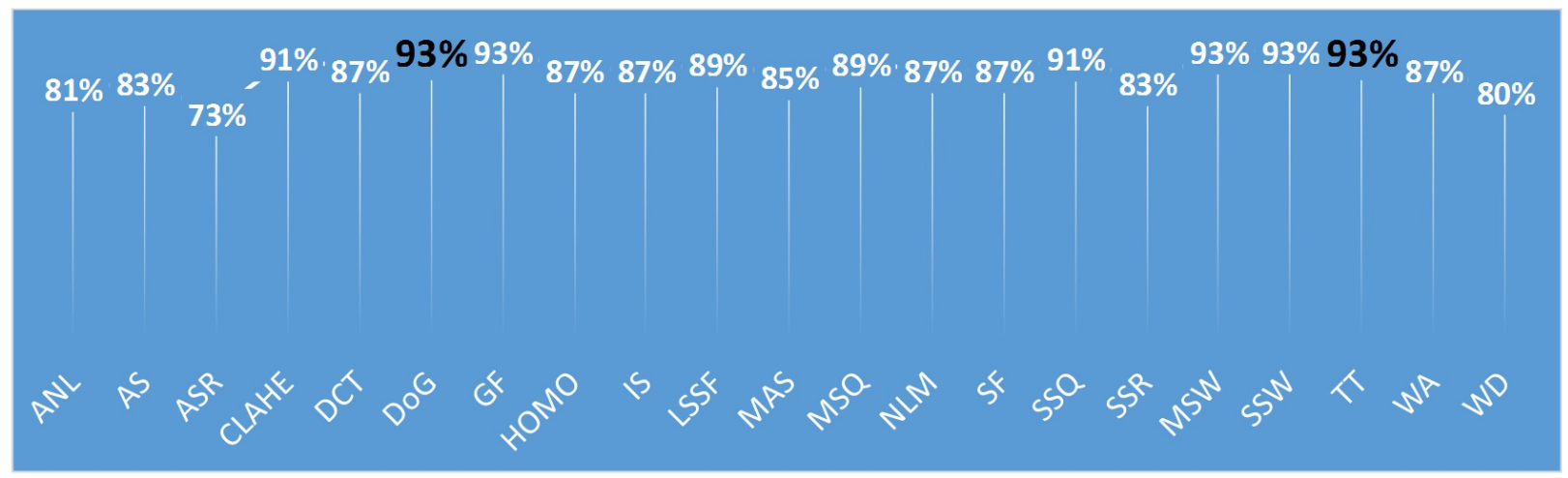

Figure 5.4: The results for $1200 \mathrm{~nm}$ vs. gallery tattoo matching applied for each of the 21 photometric normalization techniques.

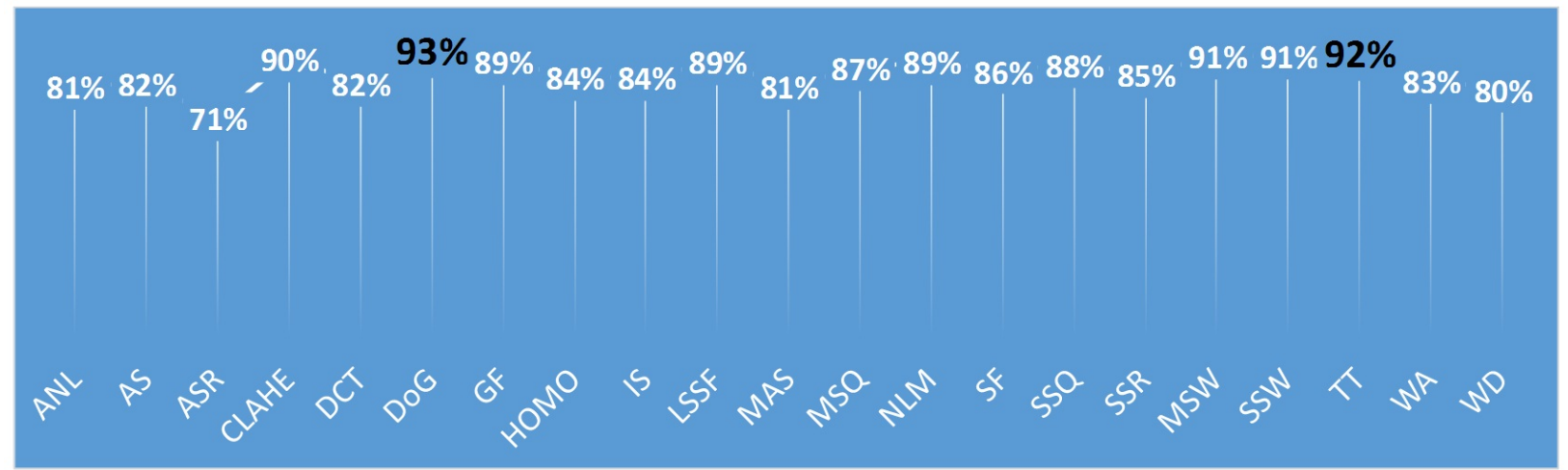

Figure 5.5: The results for $1300 \mathrm{~nm}$ vs. gallery tattoo matching applied for each of the 21 photometric normalization techniques. 


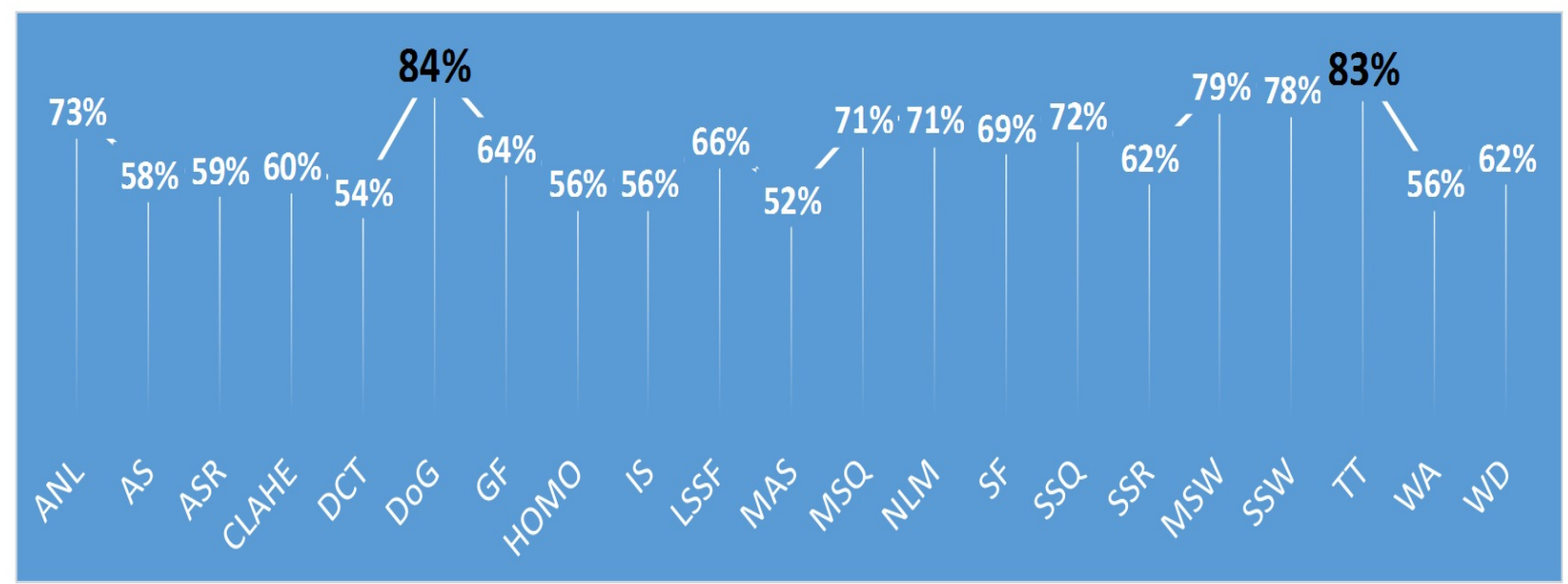

Figure 5.6: The results for $1400 \mathrm{~nm}$ vs. gallery tattoo matching applied for each of the 21 photometric normalization techniques.

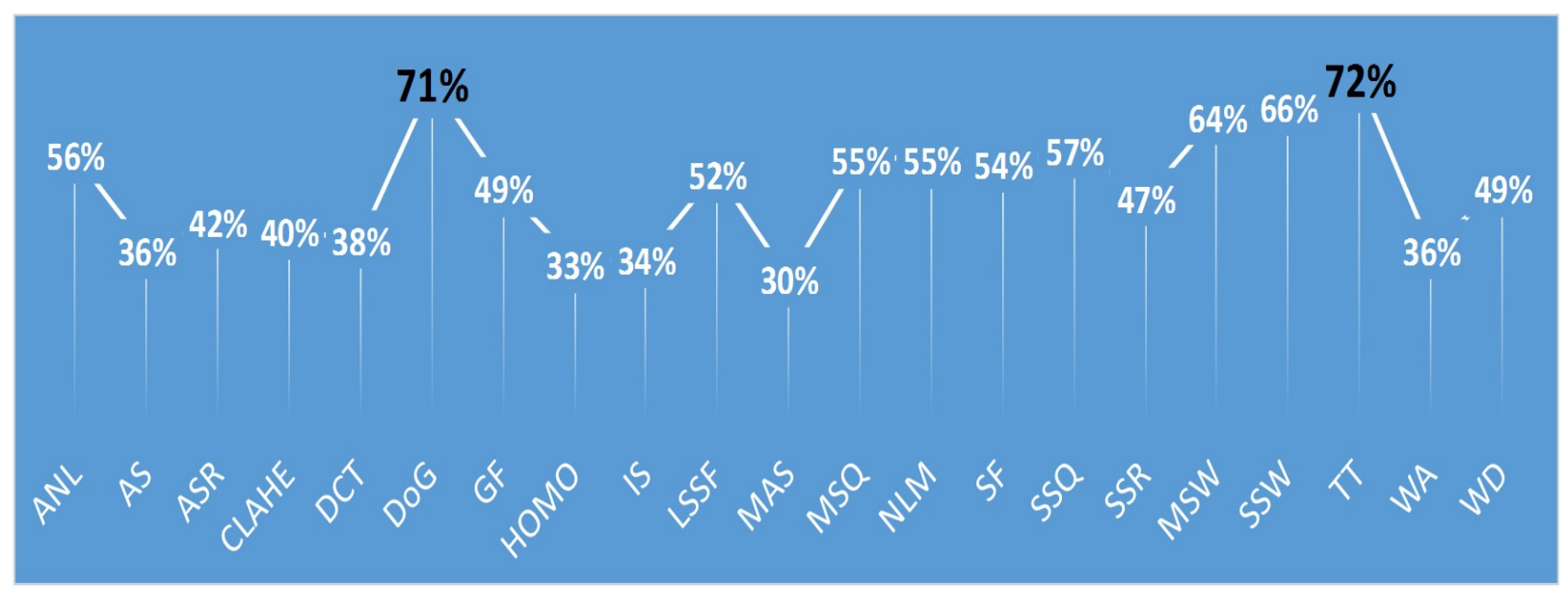

Figure 5.7: The results for $1500 \mathrm{~nm}$ vs. gallery tattoo matching applied for each of the 21 photometric normalization techniques. 


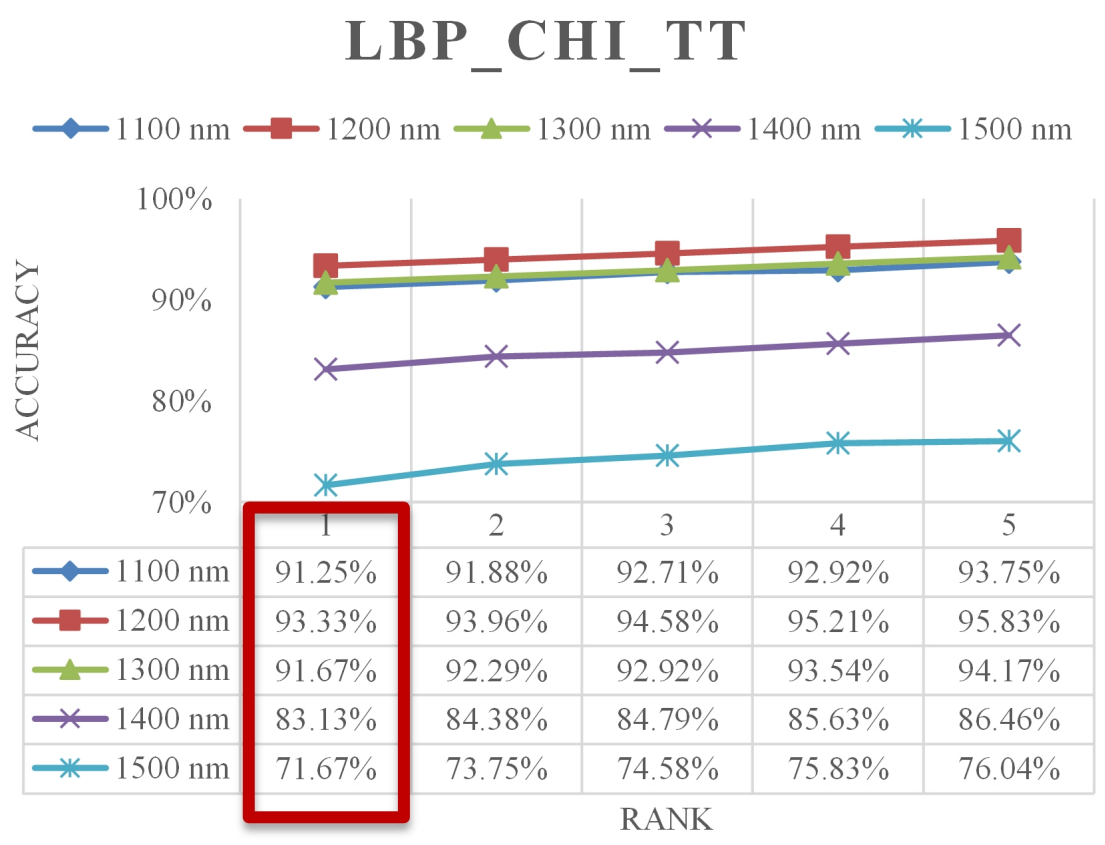

Figure 5.8: The results for all $1100 \mathrm{~nm}$ to $1500 \mathrm{~nm}$ vs. gallery tattoo matching applied for TT photometric normalization technique.

LBP_CHI_DOG

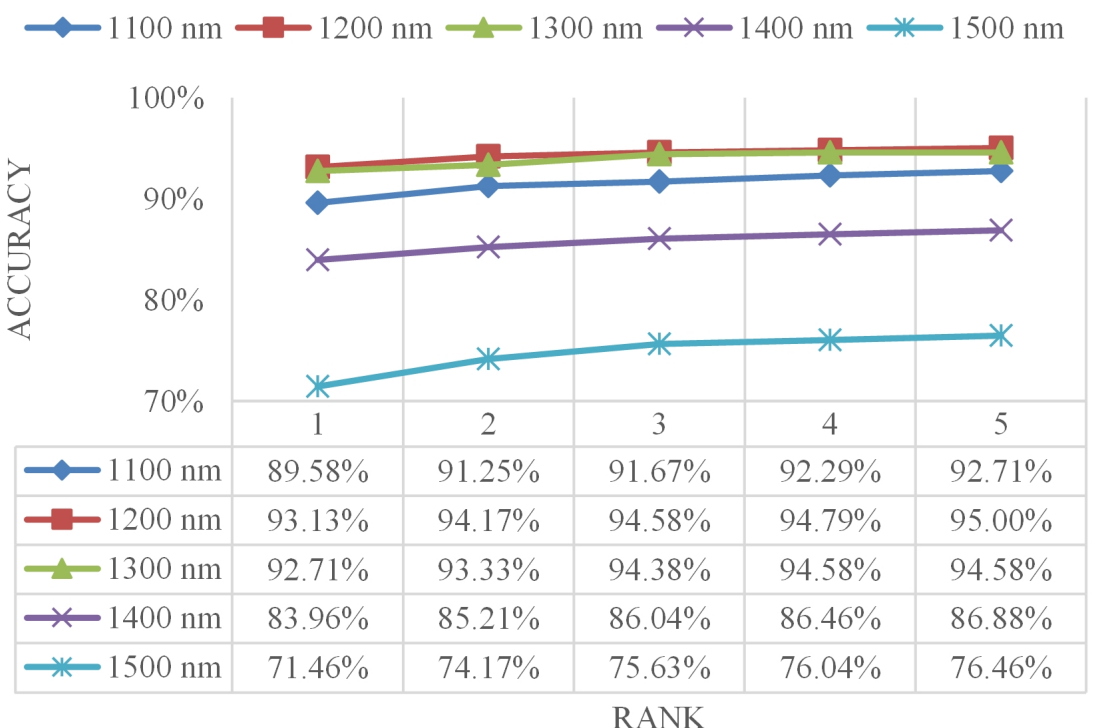

Figure 5.9: The results for all $1100 \mathrm{~nm}$ to $1500 \mathrm{~nm}$ vs. gallery tattoo matching applied for DoG photometric normalization technique. 


\begin{tabular}{|l||c|c|c|c|c|}
\hline & $1100 \mathrm{~nm}$ & $1200 \mathrm{~nm}$ & $1300 \mathrm{~nm}$ & $1400 \mathrm{~nm}$ & $1500 \mathrm{~nm}$ \\
\hline \hline Original & $83.75 \%$ & $90.63 \%$ & $89.38 \%$ & $57.92 \%$ & $37.08 \%$ \\
\hline TT & $\mathbf{9 1 . 2 5 \%}$ & $\mathbf{9 3 . 3 3 \%}$ & $91.67 \%$ & $83.13 \%$ & $\mathbf{7 1 . 6 7 \%}$ \\
\hline DoG & $89.58 \%$ & $93.13 \%$ & $\mathbf{9 2 . 7 1 \%}$ & $\mathbf{8 3 . 9 6 \%}$ & $71.46 \%$ \\
\hline
\end{tabular}

Table 5.1: Summarized of cross-spectral matching results of each band with or without applying photometric normalization technique.

results of TT and row 3 shows the results of DoG. For the band of $1100 \mathrm{~nm}$ to $1300 \mathrm{~nm}$, there are several percent accuracy increased by using photometric normalization techniques. For the band of $1400 \mathrm{~nm}$ and $1500 \mathrm{~nm}$, the accuracy has been improved tremendously (increasing about 26\% for $1400 \mathrm{~nm}$ and about $34 \%$ for $1500 \mathrm{~nm}$ ).

\subsection{Intra-spectral Tattoo Image Matching}

The matching strategy can be summarized as follow:

- $1100 \mathrm{~nm}-1500 \mathrm{~nm}$ matching with visible set.

- $1200 \mathrm{~nm}-1500 \mathrm{~nm}$ matching with $1100 \mathrm{~nm}$.

- $1300 \mathrm{~nm}-1500 \mathrm{~nm}$ matching with $1200 \mathrm{~nm}$.

- $1400 \mathrm{~nm}-1500 \mathrm{~nm}$ matching with $1300 \mathrm{~nm}$.

The CMC curves are shown in Fig. 5.10. From the results, we can find out that the intraspectral tattoo matching results are better than cross-spectral tattoo matching results for every SWIR band we used. And three of all the results can achieve $100 \%$, they are $1200 \mathrm{~nm}$ matched with $1100 \mathrm{~nm}, 1300 \mathrm{~nm}$ matched with $1100 \mathrm{~nm}$ and $1300 \mathrm{~nm}$ matched with $1200 \mathrm{~nm}$. The results of this experiment indicate two-fold: 1) the matching performance of tattoo recognition in the same spectrum (in this work is in SWIR band) is better than cross-spectral tattoo recognition; 2) the two SWIR bands are more closed to each other, the better results can be achieved (See the curve trend of $1500 \mathrm{~nm}$ line in Fig. 5.10). 


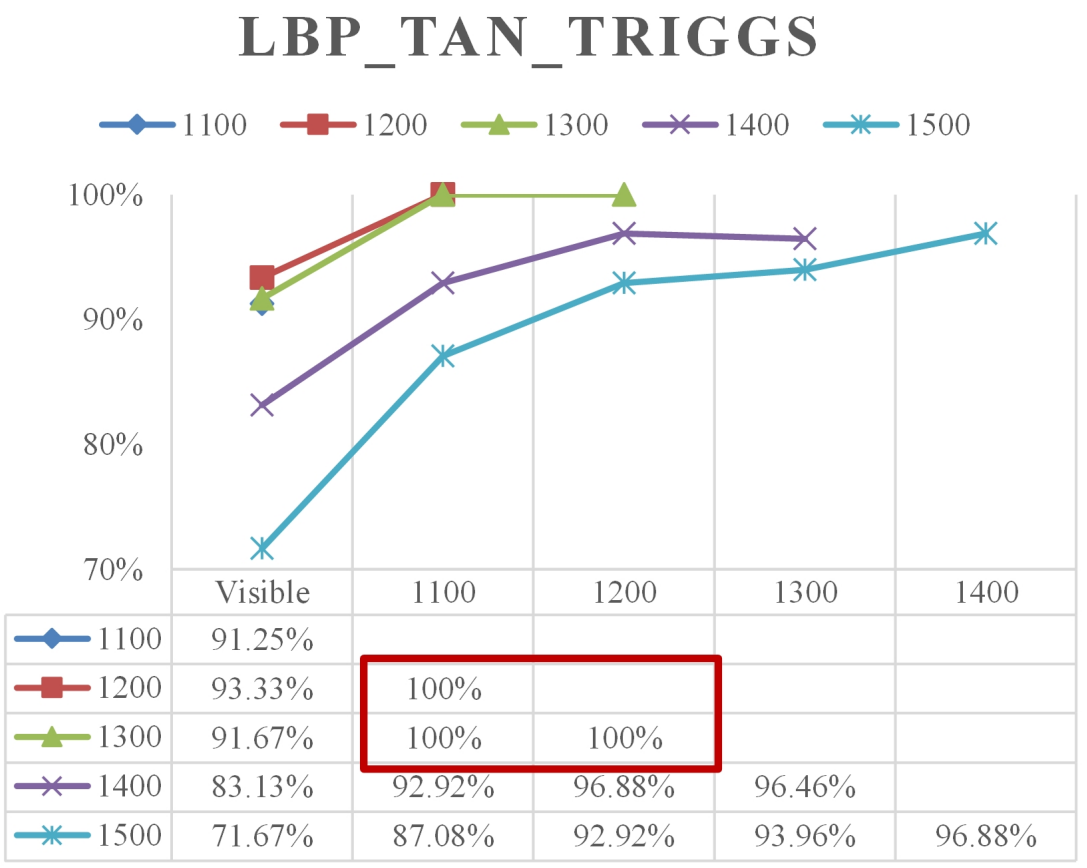

Figure 5.10: The results for inter and intra-spectral tattoo matching applied for TT photometric normalization technique.

\begin{tabular}{|l||c|c|}
\hline Database & Level-one & Level-two \\
\hline \hline NIST-Tatt-C & $94.50 \%$ & $\mathbf{1 0 0 \%}$ \\
\hline WVU-Tatt & $96.20 \%$ & $\mathbf{1 0 0 \%}$ \\
\hline
\end{tabular}

Table 5.2: Results of tattoo registration accuracy including both NIST-Tatt-C and WVU-Tatt database.

\subsection{Tattoo Registration Results}

In this section, we implemented three experiments to evaluate our proposed tattoo registration system. In the first experiment, we explored the performance of level one registration system. Both NIST-Tatt-C and WVU-Tatt databases were used to test the validity of system through SIFT descriptors and the RANSAC algorithm with a homography model for each pair of tattoos. The column of level one in Table 5.2 shows the registration results of this experiment. The accuracy of NIST-Tatt-C is $94.50 \%$ that means 6 out of 109 subjects are registered incorrectly. In the practical operation, our system filtered out 8 pairs of tattoo not registered correctly in total. However, by 


\begin{tabular}{|l||c|c|c|}
\hline Database & Original & Registration & Correction \\
\hline \hline NIST-Tatt-C & $54.13 \%$ & $95.41 \%$ & $\mathbf{1 0 0 \%}$ \\
\hline WVU-Tatt & $86.08 \%$ & $96.20 \%$ & $\mathbf{9 8 . 7 3 \%}$ \\
\hline
\end{tabular}

Table 5.3: Rank-1 of tattoo recognition performance of NIST-Tatt-C and WVU-Tatt Database based on LBP method with uniform patterns.

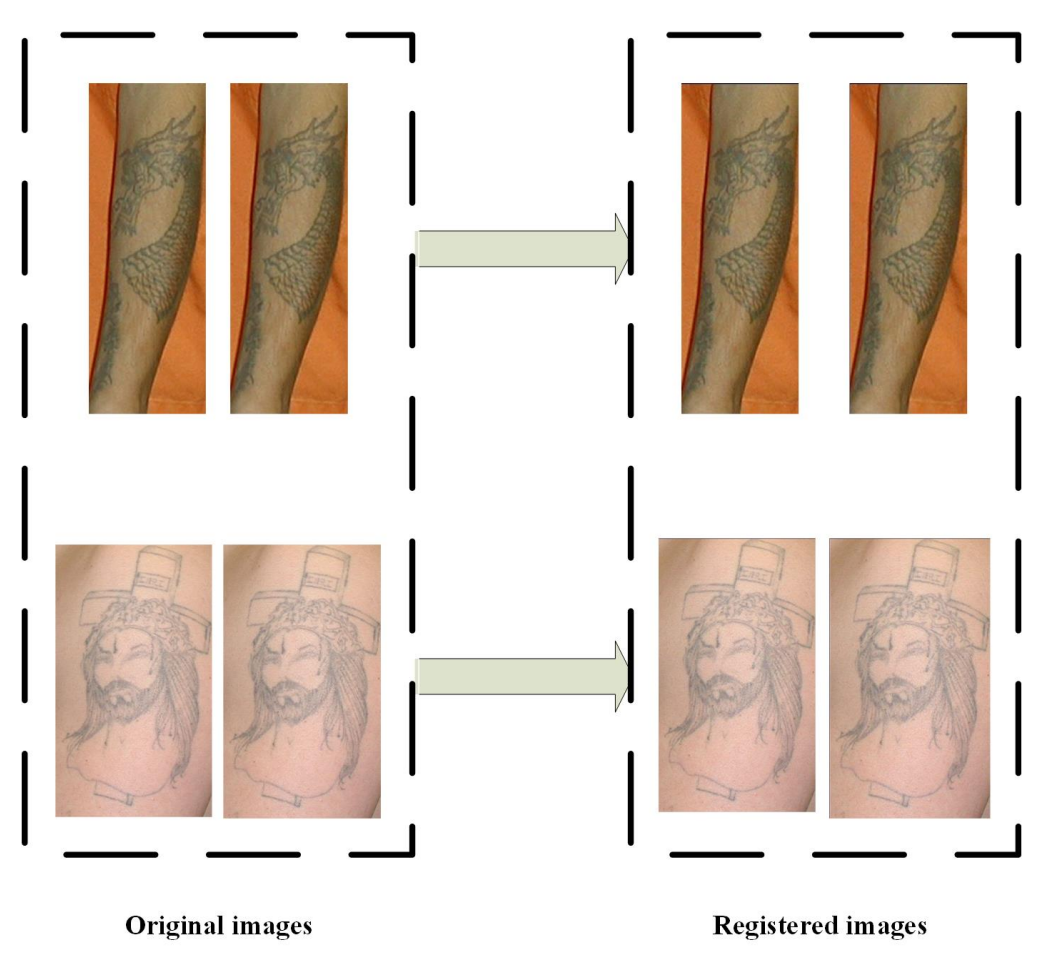

Figure 5.11: The example for already registered pair of tattoos 


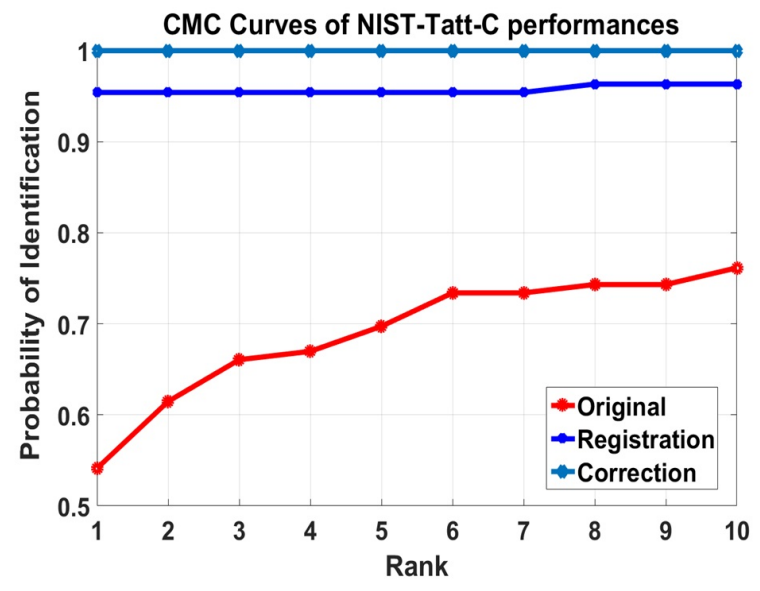

(a)

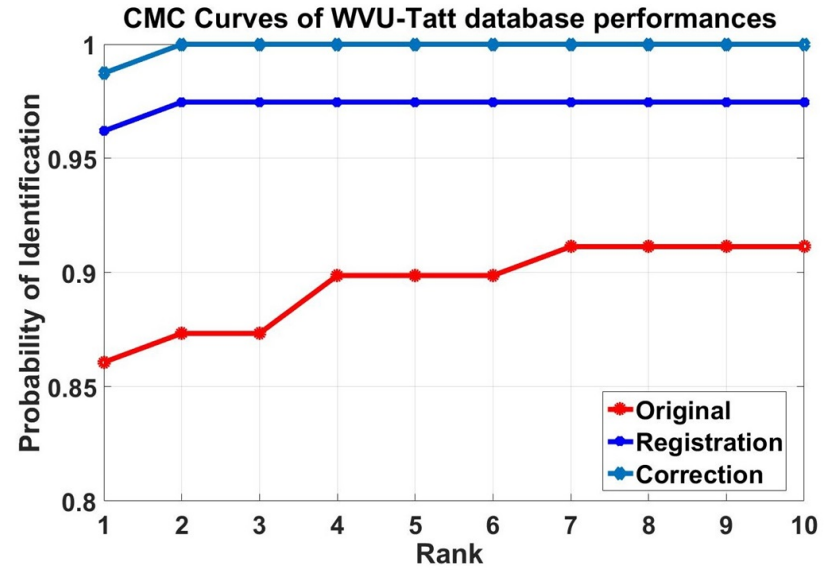

(b)

Figure 5.12: CMC curves of tattoo recognition performance based on LBP with uniform patterns: (a) indicates the results of NIST-Tatt-C database; (b) indicates the results of WVU-Tatt database.

analyzing each of incorrect registration cases specifically, there are 2 of 8 subjects that actually have already been registered with each other (see Fig. 5.11). This can account for two errors in our evaluation systems, when the system made wrong decision after the whole process finished for this 2 specific subjects. For WVU-Tatt database that has 79 subjects, the accuracy of level one is $96.20 \%$ that means 3 out of 79 subjects cannot be registered successfully. Then, all of falsely registered subjects in both databases from the first experiment are input to level two system where red-channel based CLAHE method are applied for all tattoo images first, and then applying the same process (SIFT and RANSAC with a homography model) to register again with each other. After implementing the whole system to address all the tattoo images, the final registration performance can achieve $100 \%$ for both NIST-Tatt-C and WVU-Tatt databases (refer to the column of level two in Table 5.2). Finally, LBP with uniform patterns, based on Chi square distance measurement, is used as the tattoo recognition method to compare before and after registration applied for the tattoo images. Three sub-experiments in tattoo recognition are conducted to: 1) Original: tattoo recognition without implementing any registration approach; 2) Registration: tattoo recognition with registered tattoos based on level one of system; 3) Correction: Registered tattoo recognition based on the whole registration system (implemented level one and level two of system for registration). Table 5.3 shows all of the Rank-1 performance based on these three experiments for both databases. From the results, our proposed tattoo registration system has the best 
recognition accuracy (NIST-Tatt-C is 100\%, improved $45.87 \%$ and WVU-Tatt database is $98.73 \%$, improved 12.65\%). The CMC curves in Fig. 5.12 show the LBP recognition performance from Rank-1 to Rank-10 for all three LBP based tattoo recognition experiments. 


\section{Chapter 6}

\section{Conclusions and Future Work}

In the final chapter, we summarize the conclusions of this thesis and make the general discussion for the results which we got. By analyzing this work and results, we also present the limitation of this thesis and the future plan to further improve this work.

The study and results that have been done by this work include: exploring a new area of cross-spectral matching for visible and SWIR tattoo matching; a comparative study for 21 photometric normalization techniques as pre-processing step for tattoo cross-spectral matching; the intra-spectral tattoo matching in SWIR band associating with selected photometric normalization method; and the tattoo registration system for visible band tattoo images. The limitation and future work present some important research topics for tattoo biometrics, such as automated tattoo image segmentation and localization.

\subsection{Discussion and Conclusions}

In this work, we have done the cross-spectral matching for visible against SWIR bands of tattoos and used two different databases to test the developed tattoo registration system. By our best knowledge, this is the first time to carry out tattoos captured in SWIR bands to match with both visible and SWIR bands tattoo images, also this is the first time to investigate the automatic tattoo image registration system in visible band. 


\subsubsection{Cross-Spectral Tattoo Matching}

In the experiments of cross-spectral matching problem, we firstly applied two famous descriptors to extract features from visible and SWIR tattoo images, they are SIFT which has achieved some successful results for tattoo image retrieval and matching in visible band (see the related work in Chapter 2), and $\mathrm{LBP}$ with uniform patterns which has been widely used in face recognition area including visible and Infrared (IR) bands. Through the empirical and comparative study of cross-spectral matching, we finally selected LBP with uniform patterns as the feature extraction method to gain the LBP descriptors for matching. For the pre-processing step, we compared 21 kinds of photometric normalization techniques for the tattoo cross-spectral matching and finally selected TT as the pre-processing method to apply for all tattoo images before feature extraction and matching since the approach of TT shows the most reliable results for SWIR bands of tattoo images matching with visible band. Finally, we have done the intra-spectral matching for all SWIR bands to evaluate the matching performance of IR tattoos against IR tattoo.

The potential reason why LBP has a better performance than SIFT is that the accuracy of SIFT based tattoo image matching depends on the correct keypoints matching between probe and gallery images. From visible band to match with visible band tattoo images, the local features and contents of tattoo images are similar, the color has not been changed in the same spectrum (see Fig. 5.11). But when we are trying to match $\llbracket$ tattoo images with visible tattoo images, the color content of IR tattoo images is changed a lot (see the first row of Fig. 4.3.). As increasing the SWIR filter band (from $1100 \mathrm{~nm}$ to $1500 \mathrm{~nm}$ ), not only the color is being changed, but also the visual content information is continually losing (refer introduction for more details). Therefore, SIFT algorithm becomes harder and harder to find the correct keypoints matching for the cross-spectral tattoo images. LBP descriptor is to compare the similarity of texture, although it is influenced by content and color information changed, it is impacted not too much like SIFT algorithm. Also LBP with uniform patterns are able to enhance the LBP descriptors to adapt rotation changed. Therefore, LBP with uniform patterns approach has a better performance than SIFT algorithm for cross-spectral tattoo image matching. Another side can help us better understand the content changed is to discuss the result of intra-spectral tattoo image matching (See the results in Fig. 5.10). We can easily find that the results of intra-spectral matching are always better than cross-spectral 
matching by referring the first column of Fig. 5.10, the results of cross-spectral matching always associate with the worst matching accuracy. For instance, the accuracy of $1500 \mathrm{~nm}$ vs. visible is $71.67 \%$, but the accuracy of $1100 \mathrm{~nm}$ vs. $1500 \mathrm{~nm}$ is $87.08 \%$, the accuracy of $1200 \mathrm{~nm}$ vs. 1500 $\mathrm{nm}$ is $92.92 \%$, the accuracy of $1300 \mathrm{~nm}$ vs. $1500 \mathrm{~nm}$ is $93.96 \%$ and the accuracy of $1400 \mathrm{~nm}$ vs. $1500 \mathrm{~nm}$ has achieved $96.88 \%$. This simple example demonstrates that: 1) the more closed to between two $\llbracket$ bands, the more similar content information the tattoo images have, also a better matching result can be obtained; 2) the results of the intra-spectral matching are always better than the cross-spectral matching.

\subsubsection{Tattoo Image Registration}

In the part of tattoo image registration, we presented a two-level automatic tattoo image registration system based on SIFT descriptors. This system integrates SIFT and RANSAC with a homography model for initial image registration as level one and red-channel CLAHE for error correction as level two. Through experimental analysis (detailed in Chapter 4 and Chapter 5), our tattoo image registration indicates reliable performance for both under uncontrolled conditions (NIST-Tatt-C) and controlled conditions (WVU-Tatt) tattoo databases. The benefits of this proposed registration system are three-fold: 1) our system can register and evaluate the registration performance automatically, 2) our system can implement error correction for registration automatically, 3) through processing tattoo images in our registration system, the recognition performance can be improved significantly (refer Fig. 5.12).

The potential reason why the red-channel-CLAHE integration method is the best solution of error correction for level two based registration system is that red-channel-CLAHE method provides the most obvious color difference or color distribution for the tattoo image. Fig. 6.1 provides an example to demonstrate the reason why red-channel-CLAHE is the most advanced way for our level two of tattoo registration system. In Fig. 6.1, row 1 shows the original grayscale, red, blue and green channel tattoo images. Row 2 shows the histogram of each image at row 1 . Row 3 displays the tattoo images processed by CLAHE and row 4 shows the histogram of each image at row 3. As we know, the image histogram indicates the pixel value distribution of the digital image. From all the histograms in Fig. 6.1, the color content of red-channel-CLAHE processed image has 


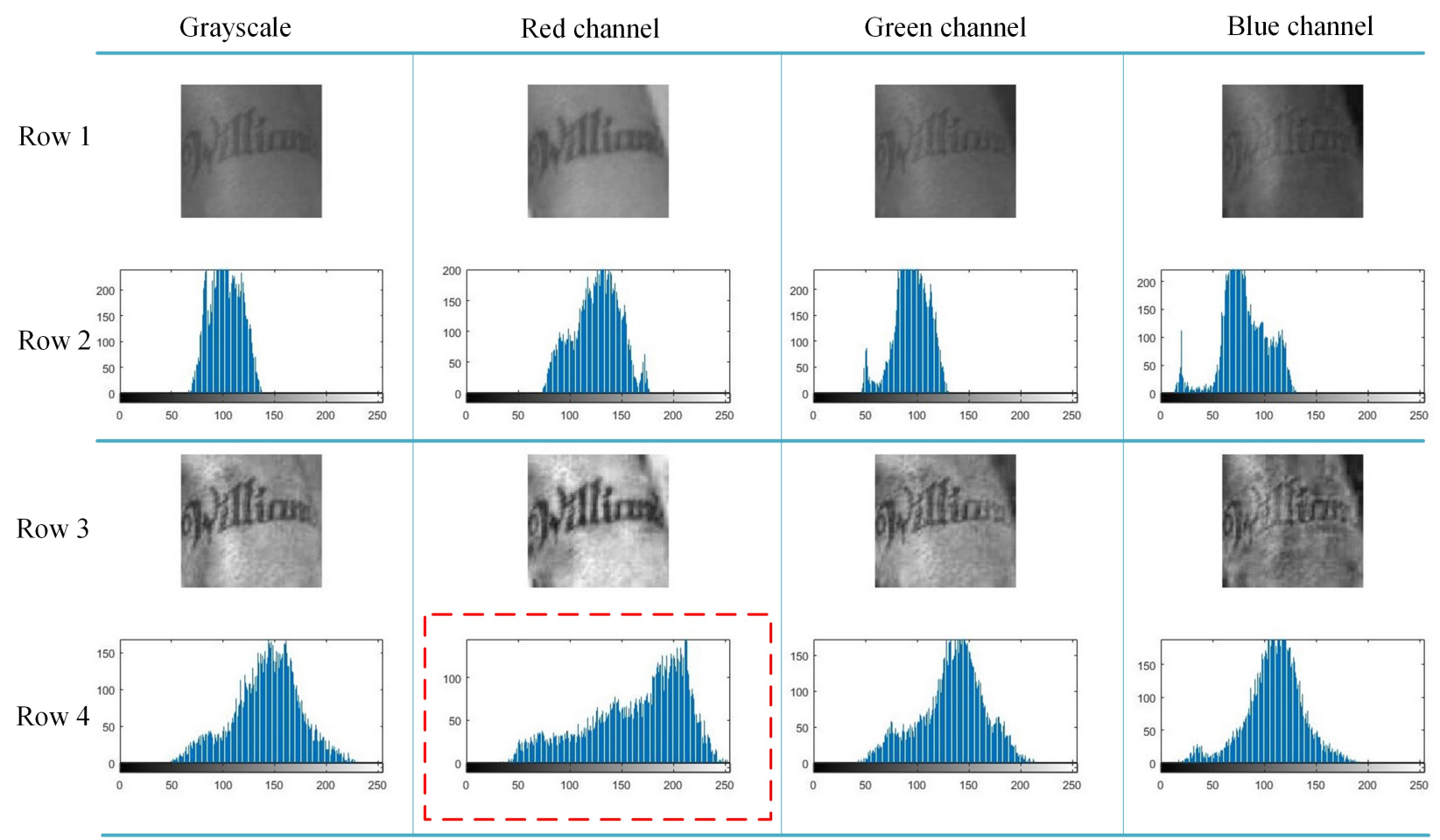

Figure 6.1: The comparative example for different color channels of tattoo processed by CLAHE or not. Row 1 shows the original grayscale, red, blue and green channel tattoo images. Row 2 shows the histograms of each of the images at row 1. Row 3 displays the tattoo images processed by CLAHE and row 4 shows the histograms of each of the images at row 3.

the widest distribution, that means in the red-channel-CLAHE processed tattoo image, the tattoo is maximum distinguished from skin. Therefore, the red-channel-CLAHE processed tattoo images can always find more SIFT keypoints matched than others (refer Table.4.1).

\subsection{Limitations}

In this work, especially for the database used for the approach of cross-spectral tattoo matching, the WVU-Tatt database was collected by very expensive cameras, and the resolution of the collected images is very high. This is one reason why we can achieve very good matching performance. In the real-world applications, law enforcement may provide much cheaper cameras for the surveillance systems and capture tattoo images with low resolution. There is no study related to multi-resolution based tattoo recognition. But in [79], Zou and Yuen explored face recognition with high and very low resolution and the results indicated that high resolution face recognition 
has a better matching performance than very low resolution face recognition. Therefore, the low resolution based tattoo recognition may be a potential challenge for our proposed cross-spectral matching method.

Another limitation of this work is that we only randomly selected 1 image each session per subject from the WVU-Tatt database where there are 10 images for per subject, each session, to test the proposed tattoo registration system. Even though we used high-speed cameras to capture the tattoo images (collecting 10 images about 2 secs), there are still existing small differences among these images which may influence the registration and matching performance.

Moreover, although there are many approaches related to tattoo matching and retrieval, even some of works have proposed the tattoo localization and segmentation methods (all segmentation and localization approaches are focus on visible band tattoo images, no existing approach is focus on tattoo image in SWIR band), the topics of automated tattoo localization and segmentation are still open, and have not been fully researched. The main challenge to prevent researchers to develop a fully automatic tattoo localization and segmentation method is that tattoo is a special biometric modality, unlike the other biometric modalities (face, ear, eye, and fingerprint), the shape, size and content of tattoos has no restriction. For this reason, automated localization and segmentation of tattoo images is extremely complicated. It is difficult to find out a unique feature to associate with algorithms to segment or localize the tattoos especially with an intricate background of tattoo image. One of newest works has done by Kim et al. [50] in 2016 gives a new perspective for tattoo localization and segmentation method, however, the accuracy of this new method are fair, an improved result still needs to be done to make this new method competitive.

\subsection{Future Work}

In the future work, firstly, we will re-run all experiments of cross-spectral matching with in total 150 subjects. Secondly, we will explore to localize and segment tattoo image automatically on both visible and SWIR bands. One possible technique can help us achieve this goal is deep learning. Deep learning has been proved as a very advanced approach in the fields of object detection, segmentation and recognition, especially for face modality. This inspires us a new insight to apply and adapt deep learning method for the problems of tattoo image detection, segmentation and 
matching. Thirdly, the tattoo matching for the SWIR band of $1400 \mathrm{~nm}$ and $1500 \mathrm{~nm}$ is still a big challenge in this work, even though the matching performance has been improved significantly by applying for photometric normalization technique, the final results are still not good enough. Therefore, to find a new way to enhance the local or global feature for the SWIR band of 1400 $\mathrm{nm}$ and $1500 \mathrm{~nm}$ and improve the recognition accuracy is another necessary step for this work. Fourthly, we will adapt our developed tattoo image registration system to make it can be used to register SWIR tattoo images. Finally, we will collect more SWIR band tattoo images to extend our database and explore large scale cross-spectral tattoo matching. 


\section{Bibliography}

[1] Huttriverofnz, "Hutts world." https://huttriverofnz.wordpress.com/2015/02/28/ now-we-know-why-isis-cover-their-hands-and-arms/, 2015.

[2] T. V. Wilsson, "How tattoos work." http://health.howstuffworks.com/skin-care/ beauty/skin-and-lifestyle/tattoo.htm.

[3] M. Hallas, "Using swir to delineate between soil and decaying canopy vegetation." http://www.harrisgeospatial.com/ProductsandSolutions/ CustomSolutions/TabId/2063/ArtMID/5503/ArticleID/14700/ Using-SWIR-to-Delineate-Between-Soil-and-Decaying-Canopy-Vegetation . aspx.

[4] T. Bourlai, N. Narang, B. Cukic, and L. Hornak, "On designing a swir multi-wavelength facialbased acquisition system," in SPIE Defense, Security, and Sensing, pp. 83530R-83530R, International Society for Optics and Photonics, 2012.

[5] "Mini swir jsx snapshot camera - 60 fps." http://www.sensorsinc.com/products/ detail/mini-swir-jsx-snapshot-camera-60-fps.

[6] A. Jain, P. Flynn, and A. A. Ross, Handbook of biometrics. Springer, 2008.

[7] A. K. Jain, S. C. Dass, and K. Nandakumar, "Can soft biometric traits assist user recognition?," in Defense and Security, pp. 561-572, International Society for Optics and Photonics, 2004.

[8] A. Dantcheva, C. Velardo, A. D'angelo, and J.-L. Dugelay, "Bag of soft biometrics for person identification," Multimedia Tools and Applications, vol. 51, no. 2, pp. 739-777, 2011.

[9] H. T. F. Rhodes, Alphonse Bertillon, father of scientific detection. Abelard-Schuman, 1956.

[10] A. K. Jain, S. C. Dass, and K. Nandakumar, "Soft biometric traits for personal recognition systems," in Biometric authentication, pp. 731-738, Springer, 2004.

[11] D. Reid, S. Samangooei, C. Chen, M. Nixon, and A. Ross, "Soft biometrics for surveillance: an overview," Machine learning: theory and applications. Elsevier, pp. 327-352, 2013.

[12] "Next generation identification (ngi)." https://www.fbi.gov/about-us/cjis/ fingerprints_biometrics/ngi. 
[13] J.-P. Beauthier, P. Lefèvre, and E. De Valck, "The tsunami threat-research and technology: Autopsy and identification techniques," The Tsunami Threat-Research and Technology, 1, p. 714, 2011.

[14] C. "Lineberry, "Tattoos." http://www.smithsonianmag.com/history/ tattoos-144038580/, 2007.

[15] "Tattoo takeover: Three in ten americans have tattoos, and most don't stop at just one." http://www .theharrispoll.com/health-and-life/Tattoo_Takeover.html, 2016.

[16] J.-E. Lee, R. Jin, A. Jain, and W. Tong, "Image retrieval in forensics: Tattoo image database application," MultiMedia, IEEE, vol. 19, pp. 40-49, Jan 2012.

[17] D. H., "Setting proper needle depth." http://www.tattoomachineequipment.com/ proper-tattoo-needle-depth.

[18] A. Bashkatov, E. Genina, V. Kochubey, and V. Tuchin, "Optical properties of human skin, subcutaneous and mucous tissues in the wavelength range from 400 to $2000 \mathrm{~nm}$," Journal of Physics D: Applied Physics, vol. 38, no. 15, p. 2543, 2005.

[19] A. Kacalak-Rzepka, S. Bielecka-Grzela, A. Klimowicz, J. Wesołowska, and R. Maleszka, "Dry skin as a dermatological and cosmetic problem," in Annales Academiae Medicae Stetinensis, vol. 54, p. 54, 2008.

[20] D. G. Lowe, "Distinctive image features from scale-invariant keypoints," International journal of computer vision, vol. 60, no. 2, pp. 91-110, 2004.

[21] T. Ahonen, A. Hadid, and M. Pietikäinen, "Face recognition with local binary patterns," in Computer vision-eccv 2004, pp. 469-481, Springer, 2004.

[22] W. Wei, H. Jun, and T. Yiping, "Image matching for geomorphic measurement based on sift and ransac methods," in Computer Science and Software Engineering, 2008 International Conference on, vol. 2, pp. 317-320, IEEE, 2008.

[23] Z. Wang and A. C. Bovik, "A universal image quality index," IEEE signal processing letters, vol. 9 , no. 3, pp. 81-84, 2002.

[24] L. Zhang, L. Zhang, X. Mou, and D. Zhang, "Fsim: a feature similarity index for image quality assessment," IEEE transactions on Image Processing, vol. 20, no. 8, pp. 2378-2386, 2011.

[25] K. Zuiderveld, "Contrast limited adaptive histogram equalization," in Graphics gems IV, pp. 474-485, Academic Press Professional, Inc., 1994.

[26] A. K. Jain, J.-E. Lee, and R. Jin, "Tattoo-id: Automatic tattoo image retrieval for suspect and victim identification," in Advances in Multimedia Information Processing-PCM 2007, pp. 256-265, Springer, 2007.

[27] F. Long, H. Zhang, and D. D. Feng, "Fundamentals of content-based image retrieval," in Multimedia Information Retrieval and Management, pp. 1-26, Springer, 2003. 
[28] J. Huang, S. R. Kumar, M. Mitra, W.-J. Zhu, and R. Zabih, "Image indexing using color correlograms," in Computer Vision and Pattern Recognition, 1997. Proceedings., 1997 IEEE Computer Society Conference on, pp. 762-768, IEEE, 1997.

[29] A. K. Jain and A. Vailaya, "Shape-based retrieval: A case study with trademark image databases," Pattern recognition, vol. 31, no. 9, pp. 1369-1390, 1998.

[30] M. J. Swain and D. H. Ballard, "Indexing via color histograms," in Active Perception and Robot Vision, pp. 261-273, Springer, 1992.

[31] J.-E. Lee, A. K. Jain, and R. Jin, "Scars, marks and tattoos (smt): Soft biometric for suspect and victim identification," in Biometrics Symposium, 2008. BSYM'08, pp. 1-8, IEEE, 2008.

[32] A. K. Jain, J.-E. Lee, R. Jin, and N. Gregg, "Content-based image retrieval: An application to tattoo images," in Image Processing (ICIP), 2009 16th IEEE International Conference on, pp. 2745-2748, IEEE, 2009.

[33] "Ansi/nist-itl 1-2000, data format for the interchange of fingerprint, facial, scar mark and tattoo (smt).." http://www.itl.nist.gov/ANSIASD/sp500-245-a16.pdf.

[34] J.-E. Lee, R. Jin, and A. Jain, "Unsupervised ensemble ranking: Application to large-scale image retrieval," in Pattern Recognition (ICPR), 2010 20th International Conference on, pp. 3902-3906, Aug 2010.

[35] J. Sivic and A. Zisserman, "Video google: A text retrieval approach to object matching in videos," in Computer Vision, 2003. Proceedings. Ninth IEEE International Conference on, pp. 1470-1477, IEEE, 2003.

[36] J.-E. Lee, R. Jin, A. K. Jain, and W. Tong, "Image retrieval in forensics: tattoo image database application," MultiMedia, IEEE, vol. 19, no. 1, pp. 40-49, 2012.

[37] J. Kim, A. Parra, J. Yue, H. Li, and E. J. Delp, "Robust local and global shape context for tattoo image matching," in Image Processing (ICIP), 2015 IEEE International Conference on, pp. 2194-2198, IEEE, 2015.

[38] J. Kim, H. Li, J. Yue, and E. J. Delp, “Tattoo image retrieval for region of interest," in Proceedings of the IEEE International Conference on Technologies for Homeland Security, 2016.

[39] E. Shechtman and M. Irani, "Matching local self-similarities across images and videos," in 2007 IEEE Conference on Computer Vision and Pattern Recognition, pp. 1-8, IEEE, 2007.

[40] H. Yi, P. Yu, X. Xu, and A. W. K. Kong, "The impact of tattoo segmentation on the performance of tattoo matching," in 2015 IEEE International WIE Conference on Electrical and Computer Engineering (WIECON-ECE), pp. 43-46, IEEE, 2015.

[41] H. Bay, T. Tuytelaars, and L. Van Gool, "Surf: Speeded up robust features," in European conference on computer vision, pp. 404-417, Springer, 2006. 
[42] M. Ngan and P. Grother, "Tattoo recognition technology-challenge (tatt-c): an open tattoo database for developing tattoo recognition research," in Identity, Security and Behavior Analysis (ISBA), 2015 IEEE International Conference on, pp. 1-6, IEEE, 2015.

[43] H. Han and A. K. Jain, "Tattoo based identification: Sketch to image matching," in 2013 International Conference on Biometrics (ICB), pp. 1-8, IEEE, 2013.

[44] J. Canny, "A computational approach to edge detection," IEEE Transactions on pattern analysis and machine intelligence, no. 6, pp. 679-698, 1986.

[45] L. Huffman and J. McDonald, "Mixed media tattoo image matching using transformed edge alignment," in Proceedings of the IEEE International Conference on Technologies for Homeland Security, 2016.

[46] S. Belongie, J. Malik, and J. Puzicha, "Shape matching and object recognition using shape contexts," IEEE transactions on pattern analysis and machine intelligence, vol. 24, no. 4, pp. 509-522, 2002.

[47] A. Myronenko and X. Song, "Point set registration: Coherent point drift," IEEE transactions on pattern analysis and machine intelligence, vol. 32, no. 12, pp. 2262-2275, 2010.

[48] P. Duangphasuk and W. Kurutach, "Tattoo skin detection and segmentation using image negative method," in Communications and Information Technologies (ISCIT), 2013 13th International Symposium on, pp. 354-359, IEEE, 2013.

[49] J. D. Allen, N. Zhao, J. Yuan, and X. Liu, "Unsupervised tattoo segmentation combining bottom-up and top-down cues," in SPIE Defense, Security, and Sensing, pp. 80630L-80630L, International Society for Optics and Photonics, 2011.

[50] J. Kim, H. Li, J. Yue, J. Ribera, E. J. Delp, and L. Huffman, “Automatic and manual tattoo localization," in Proceedings of the IEEE International Conference on Technologies for Homeland Security, 2016.

[51] J. Kim, A. Parra, H. Li, and E. J. Delp, "Efficient graph-cut tattoo segmentation," in IS\&T/SPIE Electronic Imaging, pp. $94100 \mathrm{H}-94100 \mathrm{H}$, International Society for Optics and Photonics, 2015.

[52] N. Narang and T. Bourlai, "Face recognition in the swir band when using single sensor multiwavelength imaging systems," Image and Vision Computing, vol. 33, pp. 26-43, 2015.

[53] B. Zitova and J. Flusser, "Image registration methods: a survey," Image and vision computing, vol. 21 , no. 11, pp. 977-1000, 2003.

[54] P. Dare and I. Dowman, "An improved model for automatic feature-based registration of sar and spot images," ISPRS Journal of Photogrammetry and Remote Sensing, vol. 56, no. 1, pp. 13-28, 2001.

[55] L. Yu, D. Zhang, and E.-J. Holden, "A fast and fully automatic registration approach based on point features for multi-source remote-sensing images," Computers \& Geosciences, vol. 34, no. 7 , pp. 838-848, 2008. 
[56] A. Wong and D. A. Clausi, "Aisir: Automated inter-sensor/inter-band satellite image registration using robust complex wavelet feature representations," Pattern recognition letters, vol. 31, no. 10, pp. 1160-1167, 2010.

[57] V. Štruc and N. Pavešic, "Photometric normalization techniques for illumination invariance," Advances in Face Image Analysis: Techniques and Technologies, pp. 279-300, 2011.

[58] V. Štruc and N. Pavešić, "Gabor-based kernel partial-least-squares discrimination features for face recognition," Informatica, vol. 20, no. 1, pp. 115-138, 2009.

[59] D. J. Jobson, Z.-u. Rahman, G. Woodell, et al., "Properties and performance of a center/surround retinex," Image Processing, IEEE Transactions on, vol. 6, no. 3, pp. 451-462, 1997.

[60] E. H. Land and J. McCann, "Lightness and retinex theory," JOSA, vol. 61, no. 1, pp. 1-11, 1971.

[61] Y. K. Park, S. L. Park, and J. K. Kim, "Retinex method based on adaptive smoothing for illumination invariant face recognition," Signal Processing, vol. 88, no. 8, pp. 1929-1945, 2008 .

[62] H. Wang, S. Z. Li, Y. Wang, and J. Zhang, "Self quotient image for face recognition," in Image Processing, 2004. ICIP'04. 2004 International Conference on, vol. 2, pp. 1397-1400, IEEE, 2004.

[63] W. Chen, M. J. Er, and S. Wu, "Illumination compensation and normalization for robust face recognition using discrete cosine transform in logarithm domain," Systems, Man, and Cybernetics, Part B: Cybernetics, IEEE Transactions on, vol. 36, no. 2, pp. 458-466, 2006.

[64] S. Du and R. Ward, "Wavelet-based illumination normalization for face recognition," in Image Processing, 2005. ICIP 2005. IEEE International Conference on, vol. 2, pp. II-954, IEEE, 2005.

[65] T. Zhang, B. Fang, Y. Yuan, Y. Y. Tang, Z. Shang, D. Li, and F. Lang, "Multiscale facial structure representation for face recognition under varying illumination," Pattern Recognition, vol. 42, no. 2, pp. 251-258, 2009.

[66] G. Heusch, F. Cardinaux, and S. Marcel, "Lighting normalization algorithms for face verification," tech. rep., IDIAP, 2005.

[67] R. Gross and V. Brajovic, "An image preprocessing algorithm for illumination invariant face recognition," in Audio-and Video-Based Biometric Person Authentication, pp. 10-18, Springer, 2003.

[68] V. Štruc and N. Pavešić, "Illumination invariant face recognition by non-local smoothing," in European Workshop on Biometrics and Identity Management, pp. 1-8, Springer, 2009.

[69] R. Gross and V. Brajovic, "An image preprocessing algorithm for illumination invariant face recognition," in International Conference on Audio-and Video-Based Biometric Person Authentication, pp. 10-18, Springer, 2003. 
[70] T. Zhang, Y. Y. Tang, B. Fang, Z. Shang, and X. Liu, "Face recognition under varying illumination using gradientfaces," Image Processing, IEEE Transactions on, vol. 18, no. 11, pp. 2599-2606, 2009.

[71] B. Wang, W. Li, W. Yang, and Q. Liao, "Illumination normalization based on weber's law with application to face recognition," Signal Processing Letters, IEEE, vol. 18, no. 8, pp. 462$465,2011$.

[72] X. Xie, W.-S. Zheng, J. Lai, P. C. Yuen, and C. Y. Suen, "Normalization of face illumination based on large-and small-scale features," Image Processing, IEEE Transactions on, vol. 20, no. 7, pp. 1807-1821, 2011.

[73] X. Tan and B. Triggs, "Enhanced local texture feature sets for face recognition under difficult lighting conditions," Image Processing, IEEE Transactions on, vol. 19, no. 6, pp. 1635-1650, 2010 .

[74] T. Ojala, M. Pietikäinen, and D. Harwood, "A comparative study of texture measures with classification based on featured distributions," Pattern recognition, vol. 29, no. 1, pp. 51-59, 1996.

[75] T. Ojala, M. Pietikäinen, and T. Mäenpää, "Multiresolution gray-scale and rotation invariant texture classification with local binary patterns," Pattern Analysis and Machine Intelligence, IEEE Transactions on, vol. 24, no. 7, pp. 971-987, 2002.

[76] D. Huang, C. Shan, M. Ardabilian, Y. Wang, and L. Chen, "Local binary patterns and its application to facial image analysis: a survey," Systems, Man, and Cybernetics, Part C: Applications and Reviews, IEEE Transactions on, vol. 41, no. 6, pp. 765-781, 2011.

[77] T. Ahonen, A. Hadid, and M. Pietikainen, "Face description with local binary patterns: Application to face recognition," Pattern Analysis and Machine Intelligence, IEEE Transactions on, vol. 28, no. 12, pp. 2037-2041, 2006.

[78] A. Vedaldi and B. Fulkerson, "VLFeat: An open and portable library of computer vision algorithms." http://www.vlfeat.org/, 2008.

[79] W. W. Zou and P. C. Yuen, "Very low resolution face recognition problem," IEEE Transactions on Image Processing, vol. 21, no. 1, pp. 327-340, 2012. 